\title{
Formation of a Bridging Phosphinidene Thorium Complex
}

Andrew C. Behrle, ${ }^{1}$ Ludovic Castro, ${ }^{2}$ Laurent Maron, ${ }^{2} *$ and Justin R. Walensky ${ }^{1} *$

${ }^{1}$ Department of Chemistry, University of Missouri, Columbia, MO 65211

${ }^{2}$ Universite de Toulouse and CNRS, INSA, UPS, CNRS, UMR, UMR 5215, LPCNO, 135 Avenue de Rangueil, F31077 Toulouse (France)

Table of Contents

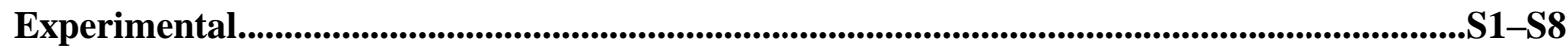

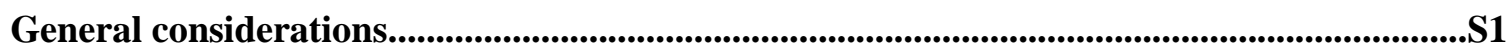

Computational details......................................................................................................................................S2

Crystallographic data collections and structure determination...................................................S3

Synthesis of 1 ...................................................................................................................................................................S3

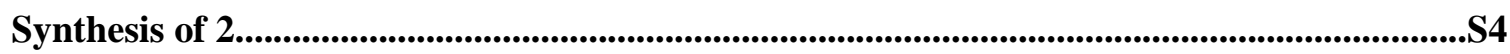

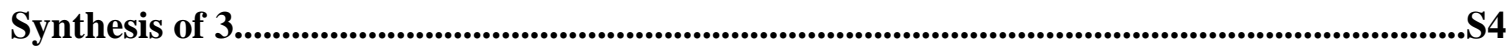

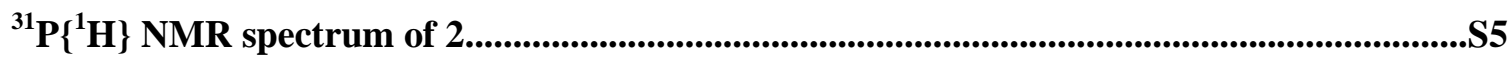

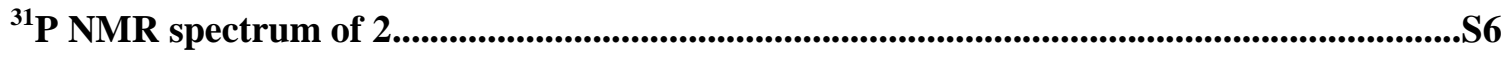

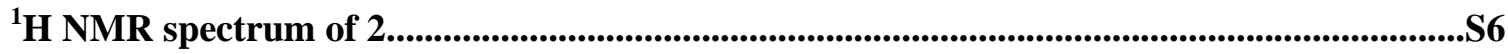

${ }^{1}$ H NMR spectrum of 2 with corresponding ChemDraw figure................................................S7

Thermal ellipsoid plot of complex 3................................................................................................................S8

X-ray crystallography data..............................................................................................................................S8

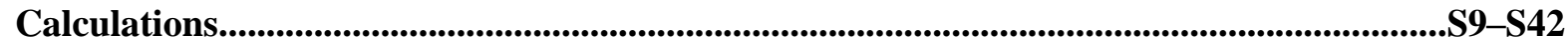

References...............................................................................................................................................................S42

\section{Experimental}

General considerations. The syntheses and manipulations described below were conducted using standard Schlenk and glovebox techniques. All reactions were conducted in a Vacuum Atmospheres inert atmosphere $\left(\mathrm{N}_{2}\right)$ glovebox or a double-manifold Schlenk line. Toluene, 1,2-dimethoxyethane, diethyl ether and hexane were purchased anhydrous, stored over activated $4 \AA$ molecular sieves, and 
sparged with nitrogen prior to use. Methylcyclohexane was dried over activated 4 Å molecular sieves and sparged with nitrogen for thirty minutes prior to use. All commercially available reactants were purchased from suppliers and used without further purification. $\left[\mathrm{ThCl}_{4}(\mathrm{DME})_{2}\right],{ }^{1}\left[\left(\mathrm{C}_{5} \mathrm{Me}_{5}\right)_{2} \mathrm{ThCl}_{2}\right],{ }^{2}$ $\left[\left(\mathrm{C}_{5} \mathrm{Me}_{5}\right)_{2} \mathrm{ThMe}_{2}\right],{ }^{2} \quad\left[\mathrm{TippPCl}_{2}\right]^{3}$ and $\left[\mathrm{H}_{2} \mathrm{PTipp}\right]^{4}$ (Tipp $\left.=2,4,6-{ }^{i} \mathrm{Pr}_{3} \mathrm{C}_{6} \mathrm{H}_{2}\right)$ were synthesized as previously described. $\mathrm{KP}(\mathrm{H})$ Tipp was made from $\mathrm{H}_{2} \mathrm{PTipp}$ and $\mathrm{K}\left[\mathrm{N}\left(\mathrm{SiMe}_{3}\right)_{2}\right]$ in toluene. Benzene- $d_{6}$ (Cambridge Isotope Laboratories) were dried over molecular sieves and degassed with three freezeevacuate-thaw cycles. All ${ }^{1} \mathrm{H}$ and ${ }^{13} \mathrm{C}$ NMR spectra were obtained on a $500 \mathrm{MHz}$ DRX Bruker spectrometer. All ${ }^{31} \mathrm{P}$ NMR spectra were obtained on a $250 \mathrm{MHz}$ ARX spectrometer at $101 \mathrm{MHz} .{ }^{1} \mathrm{H}$ NMR shifts given were referenced internally to the residual solvent peak at $\delta 7.16 \mathrm{ppm}\left(\mathrm{C}_{6} \mathrm{D}_{5} \mathrm{H}\right) .{ }^{13} \mathrm{C}$ NMR shifts given were referenced internally to the residual peak at $\delta 128.0 \mathrm{ppm}\left(\mathrm{C}_{6} \mathrm{D}_{6}\right) .{ }^{31} \mathrm{P}$ NMR spectra were externally referenced to $0.00 \mathrm{ppm}$ with $5 \% \mathrm{H}_{3} \mathrm{PO}_{4}$ in $\mathrm{D}_{2} \mathrm{O}$. Infrared spectra were recorded as $\mathrm{KBr}$ pellets on Perkin-Elmer Spectrum One FT-IR spectrometer. Elemental analysis were performed at the University of California, Berkeley Microanalytical Facility using a Perkin-Elmer Series II 2400 CHNS analyzer.

Computational details. All calculations were carried out with the Gaussian 09 suite of programs. ${ }^{5}$ Thorium atoms were treated with a 5 f-in-core effective core potential to describe their +4 oxidation state, associated with its adapted basis set. ${ }^{6}$ Carbon and hydrogen atoms were described with a $6-31 \mathrm{G}(\mathrm{d}, \mathrm{p})$ double- $\zeta$ basis set. ${ }^{7}$ Phosphorus atoms were treated with a small core relativistic effective core potential of Stuttgart. ${ }^{8}$ Calculations were carried out at the DFT level of theory with the hybrid functional B3PW91. ${ }^{9,10}$ Geometries were optimized without any symmetry restriction and the nature of the extrema was verified by analytical frequency calculations. The calculation of electronic energies and enthalpies of the extrema of the potential energy surface (minima and transition states) were performed at the same level of theory as the geometry optimizations. IRC calculations were performed to confirm the connections of the optimized transition states. The electronic charges (at the DFT level) were computed using the natural population analysis (NPA) technique. ${ }^{11}$ 
Crystallographic Data Collection and Structure Determination. The selected single crystal was mounted on nylon cryoloops using viscous hydrocarbon oil. X-ray data collection was performed at 100(2) K. The X-ray data were collected on a Bruker CCD diffractometer with monochromated Mo$\mathrm{K} \alpha$ radiation $(\lambda=0.71073 \AA)$. The data collection and processing utilized Bruker Apex 2 suite of programs. ${ }^{12}$ The structures were solved using direct methods and refined by full-matrix least-squares methods on F2 using Bruker SHELX-2014/7 program. ${ }^{13}$ All non-hydrogen atoms were refined with anisotropic displacement parameters. All hydrogen atoms were placed at calculated positions and included in the refinement using a riding model. Thermal ellipsoid plots were prepared by using Xseed $^{14}$ with $50 \%$ of probability displacements for non-hydrogen atoms.

$\left(\mathbf{C}_{5} \mathbf{M e}_{5}\right)_{2} \mathbf{T h}\left[\mathbf{P}(\mathbf{H})\right.$ Tipp $_{2}$ (1). A $20 \mathrm{~mL}$ scintillation vial was charged with $\left(\mathrm{C}_{5} \mathrm{Me}_{5}\right)_{2} \mathrm{ThCl}_{2}$ (280 mg, $0.488 \mathrm{mmol})$, toluene $\left(3 \mathrm{~mL}\right.$ ) and placed in $-25{ }^{\circ} \mathrm{C}$ freezer for 25 minutes. A second $20 \mathrm{~mL}$ scintillation vial was charged with $\mathrm{KP}(\mathrm{H}) \mathrm{Tipp}(281 \mathrm{mg}, 1.02 \mathrm{mmol})$ and toluene (2 $\mathrm{mL})$. The $\mathrm{KP}(\mathrm{H})$ Tipp mixture was added to a stirring solution of $\left(\mathrm{C}_{5} \mathrm{Me}_{5}\right)_{2} \mathrm{ThCl}_{2}$ and allowed to stir for $2 \mathrm{~h}$. The toluene was removed under vacuum to yield a vibrant orange solid which was extracted with methylcyclohexane $(2 \times 6 \mathrm{~mL})$, filtered over Celite, concentrated and placed in a $-25{ }^{\circ} \mathrm{C}$ to yield an orange solid $(285 \mathrm{mg}, 60 \%)$. X-ray quality crystals were grown from a concentrated hexane solution at room temperature. ${ }^{1} \mathrm{H}\left(\mathrm{C}_{6} \mathrm{D}_{6}, 298 \mathrm{~K}\right): \delta 7.25(\mathrm{~s}, 4 \mathrm{H}$, $\operatorname{ArH}), 3.93\left(\mathrm{~d}, 2 \mathrm{H},{ }^{1} J_{\mathrm{P}-\mathrm{H}}=225 \mathrm{~Hz}, \mathrm{PH}\right), 3.90-3.86\left(\mathrm{~m}, 4 \mathrm{H}, \mathrm{C} H_{\text {ortho }}\left(\mathrm{CH}_{3}\right)_{2}\right), 2.94\left(\mathrm{sept}, 2 \mathrm{H},{ }^{3} J_{\mathrm{H}-}\right.$ $\left.\mathrm{H}=7.0 \mathrm{~Hz}, \mathrm{CH}_{\mathrm{para}}\left(\mathrm{CH}_{3}\right)_{2}\right), 1.96\left(\mathrm{~s}, 30 \mathrm{H}, \mathrm{C}_{5} \mathrm{Me}_{5}\right), 1.54\left(\mathrm{~d}, 24 \mathrm{H},{ }^{3} J_{\mathrm{H}-\mathrm{H}}=7.0 \mathrm{~Hz}, \mathrm{CH}\left(\mathrm{CH}_{3}\right)_{2 \text { ortho }}\right)$, $1.34\left(\mathrm{~d}, 12 \mathrm{H},{ }^{3} J_{\mathrm{H}-\mathrm{H}}=7.0 \mathrm{~Hz}, \mathrm{CH}\left(\mathrm{CH}_{3}\right)_{2 \mathrm{para}}\right) .{ }^{13} \mathrm{C}\left\{{ }^{1} \mathrm{H}\right\}\left(\mathrm{C}_{6} \mathrm{D}_{6}, 298 \mathrm{~K}\right): \delta 151.23,146.75,137.32$ $\left(\mathrm{d},{ }^{1} J_{\mathrm{P}-\mathrm{C}}=18 \mathrm{~Hz}\right), 126.70,120.37,34.59,33.98\left(\mathrm{~d},{ }^{3} J_{\mathrm{P}-\mathrm{C}}=12.0 \mathrm{~Hz}\right), 24.69,24.53,11.74$. ${ }^{31} \mathrm{P}\left\{{ }^{1} \mathrm{H}\right\}\left(\mathrm{C}_{6} \mathrm{D}_{6}, 298 \mathrm{~K}\right): \delta$ 1.66. IR $\left(\mathrm{cm}^{-1}\right): 29.60(\mathrm{~s}), 2926$ (s), $2896(\mathrm{~s}), 2866(\mathrm{~s}), 2359(\mathrm{w})$, $2330(\mathrm{w}), 1625(\mathrm{w}), 1598(\mathrm{w}), 1543(\mathrm{w}), 1460(\mathrm{~s}), 1422(\mathrm{~m}), 1380(\mathrm{~s}), 1358(\mathrm{~m}), 1314(\mathrm{w})$, $1289(\mathrm{w}), 1258(\mathrm{w}), 1226(\mathrm{w}), 1164(\mathrm{~m}), 1103(\mathrm{~m}), 1062(\mathrm{~m}), 1022(\mathrm{w}), 957(\mathrm{w}), 934(\mathrm{w})$, 873 (m), 800 (w), 744 (w), 643 (w), 613 (w), 489 (w). UV-Vis $\left(4.4\right.$ x $10^{-4}$ M, 
methylcyclohexane): $445 \mathrm{~nm}\left(\varepsilon=138 \mathrm{M}^{-1} \mathrm{~cm}^{-1}\right) ; 268 \mathrm{~nm}\left(\varepsilon=8098 \mathrm{M}^{-1} \mathrm{~cm}^{-1}\right)$. Anal. calcd. for $\mathrm{C}_{50} \mathrm{H}_{78} \mathrm{P}_{2} \mathrm{Th}: \mathrm{C}, 61.71 \% ; \mathrm{H}, 8.08 \%$. Found $\mathrm{C}, 61.37 \% ; \mathrm{H}, 8.06 \%$.

$\left[\left(\mathrm{C}_{5} \mathrm{Me}_{5}\right)_{2} \mathbf{T h}\right]_{2}-\boldsymbol{\mu}_{2}-\mathrm{P}-\boldsymbol{\kappa}^{2}-\left\{\left[2,6-\left(\mathrm{CH}_{2}\right) \mathrm{CH}\left(\mathrm{CH}_{3}\right)\right]\right\}_{2}\left(4-\left(\mathrm{CH}_{3}\right)_{2} \mathrm{CH}-\mathrm{C}_{6} \mathbf{H}_{2}\right)(2)$. A $100 \mathrm{~mL}$ Schlenk flask was charged with $\left(\mathrm{C}_{5} \mathrm{Me}_{5}\right)_{2} \mathrm{ThMe}_{2}(350 \mathrm{mg}, 0.657 \mathrm{mmol})$ and toluene $(4 \mathrm{~mL})$. A $20 \mathrm{~mL}$ scintillation vial was charged with $\mathrm{H}_{2}$ PTipp $(76 \mathrm{mg}, 0.329 \mathrm{mmol})$ and toluene $(2 \mathrm{~mL})$. The $\mathrm{H}_{2}$ PTipp was added to the original reaction mixture and heated to $90-95{ }^{0} \mathrm{C}$ for $12-14 \mathrm{~h}$ to yield a red solution. The solvent was removed under vacuum and taken inside the glovebox. The red solid was dissolved with toluene $(8 \mathrm{~mL})$ and filtered over Celite, concentrated to 1-2 $\mathrm{mL}$ and placed in a $-25{ }^{0} \mathrm{C}$ freezer. An orange/red solid was recovered and dried under vacuum (256 mg, 63\%). X-ray quality crystals were grown from a concentrated toluene solution at room temperature or from a methylcyclohexane/hexane mixture at $-25{ }^{0} \mathrm{C} .{ }^{1} \mathrm{H}$ $\left(\mathrm{C}_{6} \mathrm{D}_{6}, 298 \mathrm{~K}\right): \delta 7.19(\mathrm{~s}, 2 \mathrm{H}, \mathrm{Ar} H), 3.98-3.94\left(\mathrm{~m}, 2 \mathrm{H}, \mathrm{CH}_{\text {ortho }}\left(\mathrm{CH}_{3}\right)\left(\mathrm{CH}_{2}\right)\right), 2.88\left(\mathrm{sep}, 1 \mathrm{H},{ }^{1} J_{\mathrm{H}-}\right.$ $\left.\mathrm{H}=7.0 \mathrm{~Hz}, \mathrm{CH}_{\mathrm{para}}\left(\mathrm{CH}_{3}\right)_{2}\right), 2.27\left(\mathrm{~s}, 30 \mathrm{H}, \mathrm{C}_{5} M e_{5}\right), 1.90\left(\mathrm{~s}, 30 \mathrm{H}, \mathrm{C}_{5} M e_{5}\right), 1.85\left(\mathrm{~d}, 6 \mathrm{H},{ }^{3} J_{\mathrm{H}-\mathrm{H}}=\right.$ $\left.6.0 \mathrm{~Hz}, \mathrm{CH}\left(\mathrm{CH}_{3}\right)_{\text {ortho }}\left(\mathrm{CH}_{2}\right)\right), 1.64\left(\mathrm{~d}, 2 \mathrm{H},{ }^{2} J_{\mathrm{H}-\mathrm{H}}=12.0 \mathrm{~Hz}, \mathrm{CH}\left(\mathrm{CH}_{3}\right)\left(\mathrm{CH}_{2}\right)_{\text {ortho }}\right), 1.35(\mathrm{~d}, 6 \mathrm{H}$, $\left.{ }^{3} J_{\mathrm{H}-\mathrm{H}}=7.0 \mathrm{~Hz}, \mathrm{CH}\left(\mathrm{CH}_{3}\right)_{2 \text { para }}\right), 0.71\left(\mathrm{~d}, 2 \mathrm{H},{ }^{2} J_{\mathrm{H}-\mathrm{H}}=12.0 \mathrm{~Hz}, \mathrm{CH}\left(\mathrm{CH}_{3}\right)\left(\mathrm{CH}_{2}\right)_{\text {ortho }}\right) .{ }^{13} \mathrm{C}\left\{{ }^{1} \mathrm{H}\right\}$ $\left(\mathrm{C}_{6} \mathrm{D}_{6}, 298 \mathrm{~K}\right): \delta 154.61,146.02,130.10\left(\mathrm{~d},{ }^{1} J_{\mathrm{P}-\mathrm{C}}=62 \mathrm{~Hz}\right), 124.30\left(\mathrm{C}_{5} \mathrm{Me}_{5}\right), 123.23\left(\mathrm{C}_{5} \mathrm{Me}_{5}\right)$, 78.32, $37.00\left(\mathrm{~d},{ }^{3} J_{\mathrm{P}-\mathrm{C}}=5.0 \mathrm{~Hz}\right), 34.88,30.68,24.38,12.30\left(\mathrm{C}_{5} \mathrm{Me}_{5}\right),\left(11.32\left(\mathrm{C}_{5} \mathrm{Me}_{5}\right) .{ }^{31} \mathrm{P}\left\{{ }^{1} \mathrm{H}\right\}\right.$ $\left(\mathrm{C}_{6} \mathrm{D}_{6}, 298 \mathrm{~K}\right): \delta$ 161.9. IR ( $\left.\mathrm{cm}^{-1}\right): 2959$ (s), 2907 (s), 2861 (s), 2724 (w), 1599 (m), 1442 (s), 1380 (s), 1259 (m), 1085 (s), 955 (m), 874 (m), 802 (m), 728 (m), 615 (m), 476 (m). UV-Vis (3.0 x $10^{-3} \mathrm{M}$, methylcyclohexane): $467 \mathrm{~nm}\left(\varepsilon=1348 \mathrm{M}^{-1} \mathrm{~cm}^{-1}\right)$. Anal. calcd. for $\mathrm{C}_{55} \mathrm{H}_{81} \mathrm{P}_{1} \mathrm{Th}_{2}$ : C, 53.39\%; H, 6.60\%. Found C, 53.18\%; H, $6.97 \%$.

$\left(\mathrm{C}_{5} \mathbf{M e}_{5}\right)_{2} \mathbf{T h}(\mathrm{OAr})_{2}\left(\mathrm{Ar}=\mathbf{2 , 6}-\mathrm{Me}_{2} \mathbf{C}_{6} \mathbf{H}_{3}\right)(3)$. A $20 \mathrm{~mL}$ scintillation vial was charged with 2 (180 mg, $0.185 \mathrm{mmol})$ and methylcyclohexane $(4 \mathrm{~mL})$. The vial was placed in $-25{ }^{\circ} \mathrm{C}$ freezer for 20 minutes. A second $20 \mathrm{~mL}$ scintillation vial was charged with $\mathrm{HOAr}(\mathrm{Ar}=2,6-$ $\left.\mathrm{Me}_{2} \mathrm{C}_{6} \mathrm{H}_{3}\right)(45 \mathrm{mg}, 0.368 \mathrm{~mol})$ and methylcyclohexane $(1 \mathrm{~mL})$. The HOAr mixture was added 
to the initial reaction mixture resulting in color change to pale yellow. The reaction was monitored by ${ }^{31} \mathrm{P}$ NMR spectroscopy and complete after $4 \mathrm{~h}$ at room temperature. The solvent was removed under vacuum to yield a colorless solid. The solid was extracted with diethyl ether, filtered over Celite, concentrated and placed in a $-25{ }^{\circ} \mathrm{C}$ freezer. X-ray quality crystals were grown from a concentrated diethyl ether solution at $-25{ }^{\circ} \mathrm{C}(100 \mathrm{mg}, 73 \%) .{ }^{1} \mathrm{H}\left(\mathrm{C}_{6} \mathrm{D}_{6}\right.$, 298K): $\delta 7.11\left(\mathrm{~d}, 2 \mathrm{H},{ }^{3} J_{\mathrm{H}-\mathrm{H}}=7.0 \mathrm{~Hz}, \operatorname{Ar} H_{\text {meta }}\right), 7.05\left(\mathrm{~d}, 2 \mathrm{H},{ }^{3} J_{\mathrm{H}-\mathrm{H}}=7.0 \mathrm{~Hz}, \operatorname{Ar} H_{\text {meta }}\right), 6.81(\mathrm{t}$, $\left.2 \mathrm{H},{ }^{3} J_{\mathrm{H}-\mathrm{H}}=7.0 \mathrm{~Hz}, \mathrm{Ar} H_{\mathrm{para}}\right), 2.44\left(\mathrm{~s}, 6 \mathrm{H}, 2,6-M e_{2} \mathrm{C}_{6} \mathrm{H}_{3}\right), 2.31\left(\mathrm{~s}, 6 \mathrm{H}, 2,6-M e_{2} \mathrm{C}_{6} \mathrm{H}_{3}\right), 1.94(\mathrm{~s}$, $\left.30 \mathrm{H}, \mathrm{C}_{5} M e_{5}\right) .{ }^{13} \mathrm{C}\left\{{ }^{1} \mathrm{H}\right\}\left(\mathrm{C}_{6} \mathrm{D}_{6}, 298 \mathrm{~K}\right): \delta 160.94,129.03,128.47,126.99,125.91,125.80$, 119.44, 19.65, 19.24, 11.37. IR ( $\left.\mathrm{cm}^{-1}\right): 2965$ (m), 2910 (s), 2864 (s), 1590 (m), 1457 (s), 1268 (s), 1218 (s), 1092 (m), 1024 (w), 864 (s), 756 (m), 707 (m), 535 (m), 476 (m). Anal. calcd. for $\mathrm{C}_{36} \mathrm{H}_{48} \mathrm{O}_{2}$ Th Th: C, 58.05\%; H, 6.50\%. Found C, 58.30\%; H, 6.26\%.

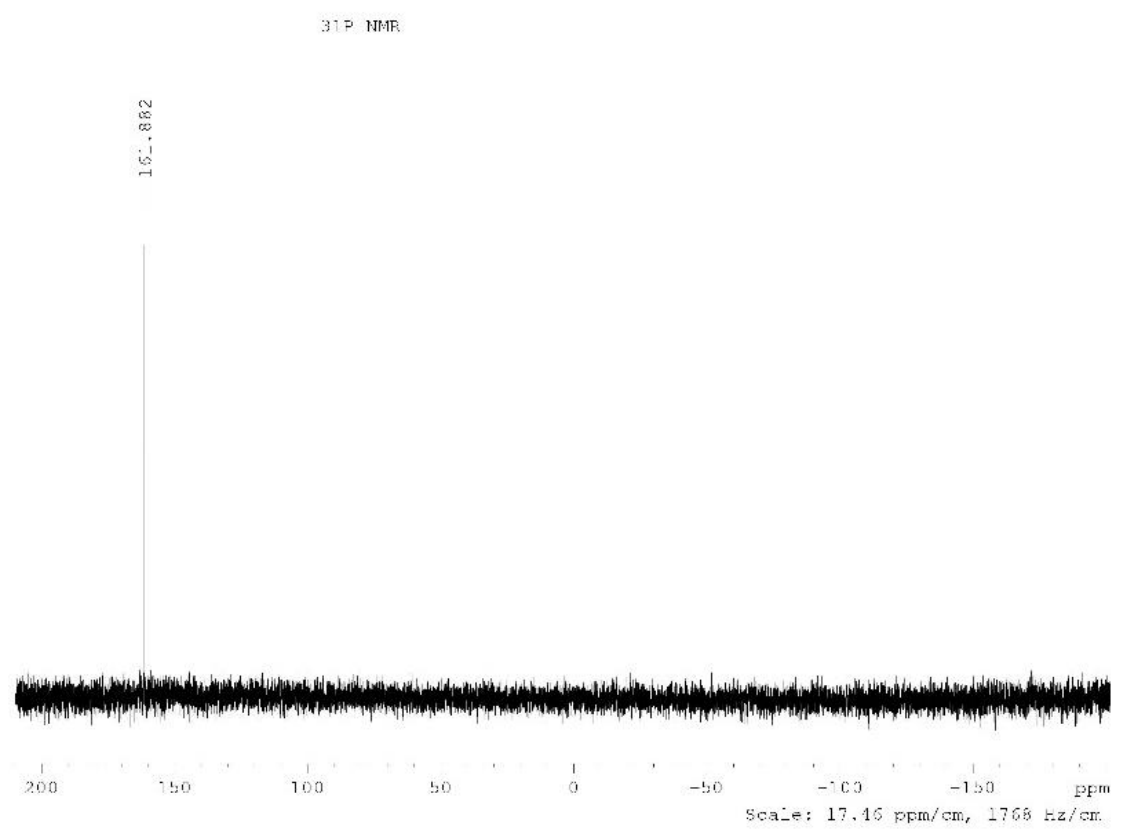

Figure S1. ${ }^{31} \mathrm{P}\left\{{ }^{1} \mathrm{H}\right\}$ NMR spectra of compound 2 showing resonance at $161.9 \mathrm{ppm}$. 


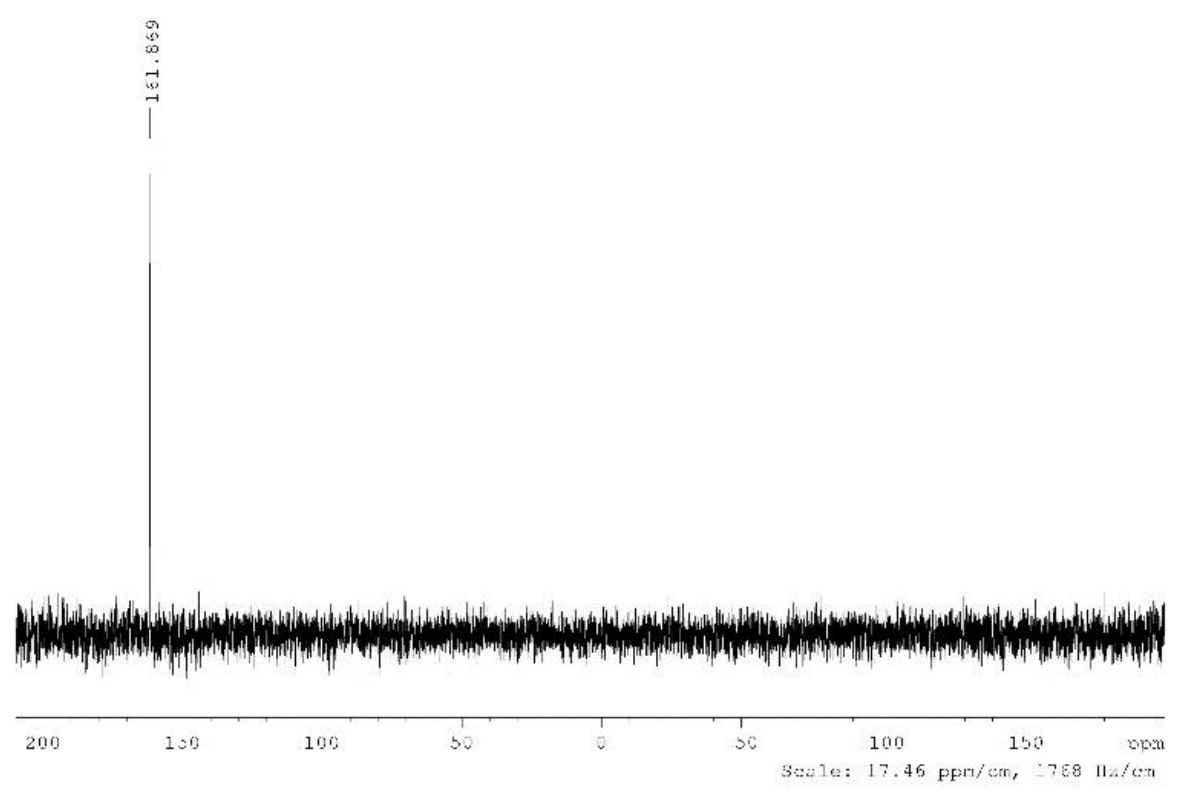

Figure S2. ${ }^{31} \mathrm{P}$ NMR spectra of compound 2 showing resonance at $161.9 \mathrm{ppm}$.

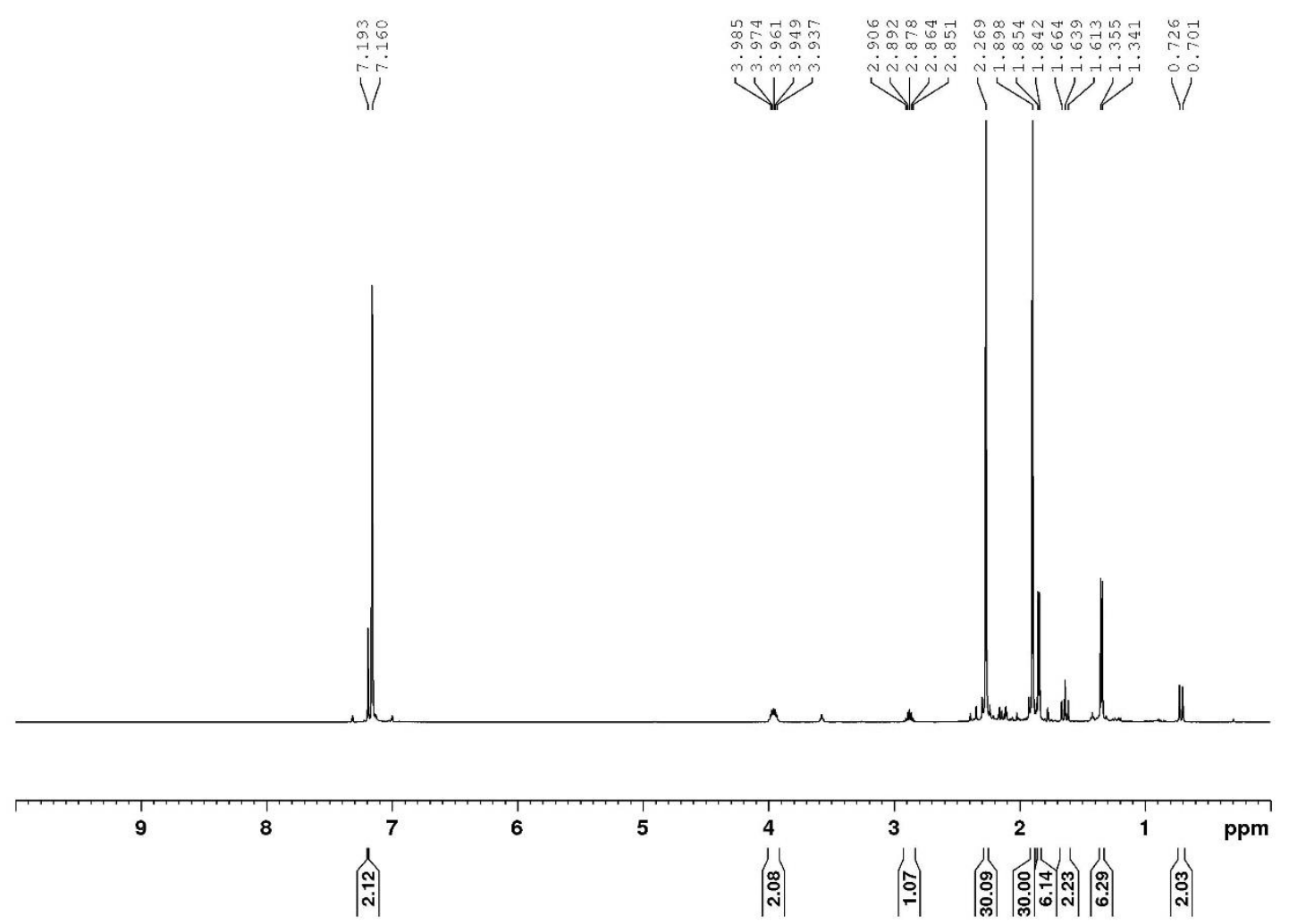

Figure S3. Full ${ }^{1} \mathrm{H}$ NMR spectrum of compound 2. 


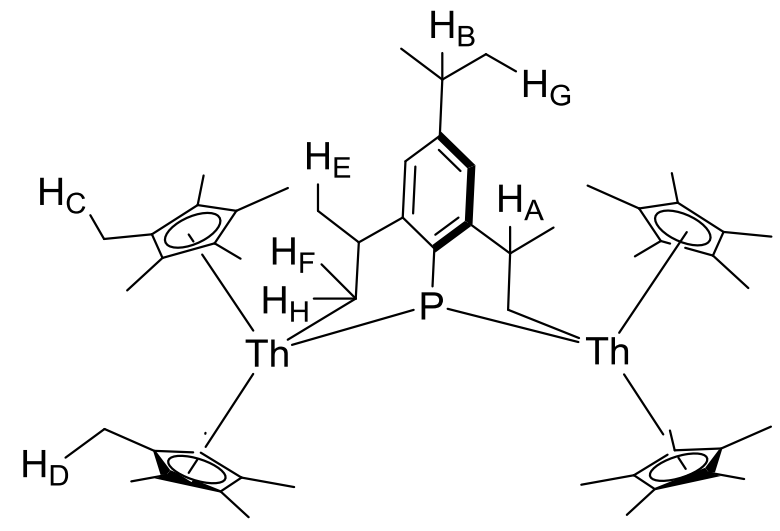

1
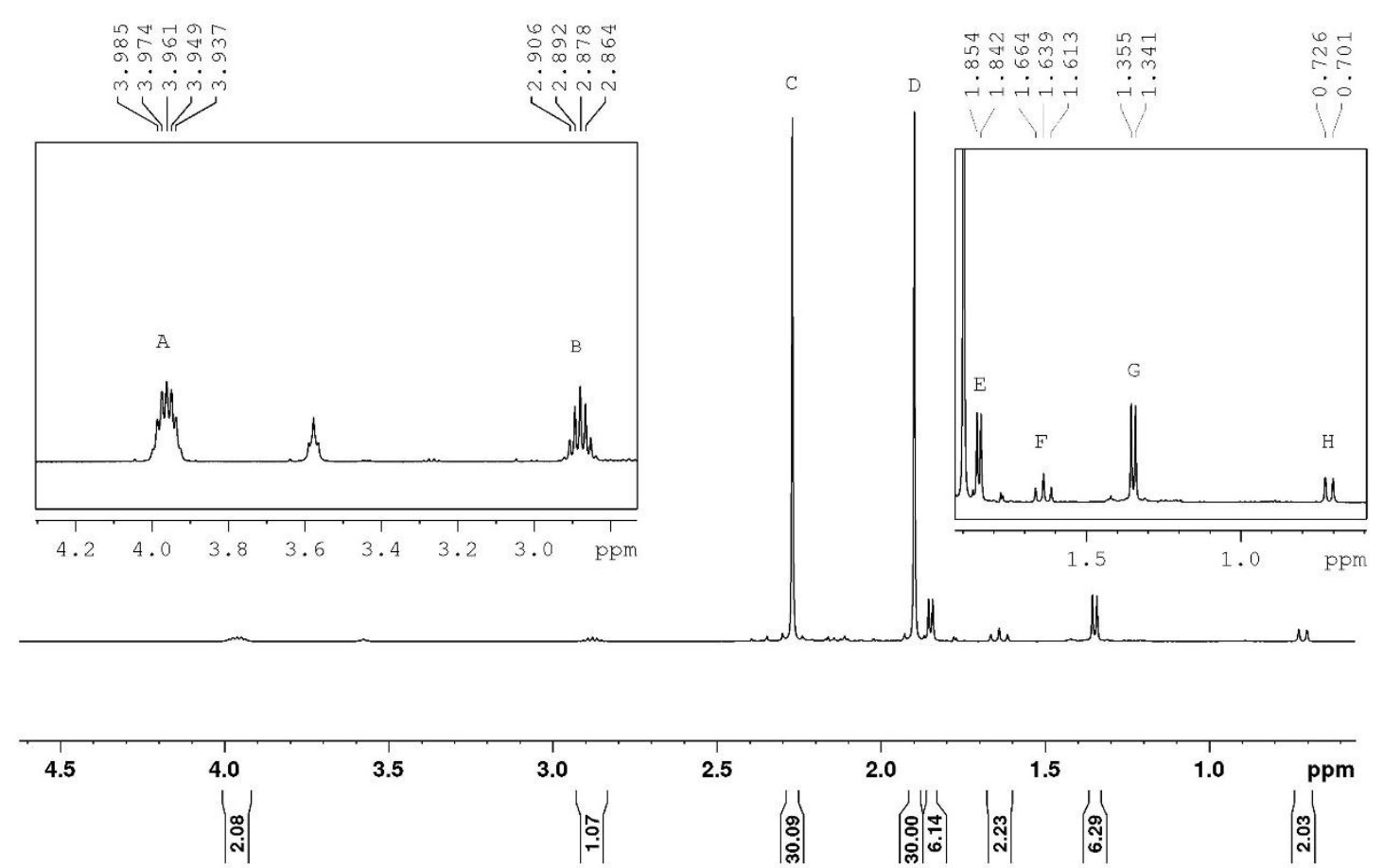

Figure S4. ${ }^{1} \mathrm{H}$ NMR spectrum of compound 2 with labeled resonances and ChemDraw figure. 


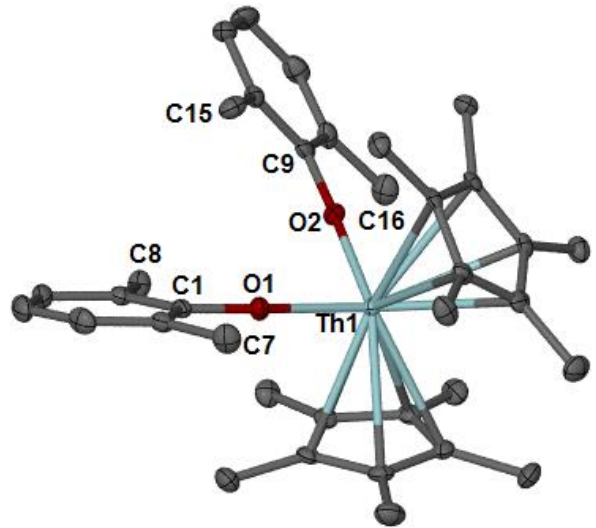

Figure S5. Thermal ellipsoid plot of $\mathbf{3}$ shown at the 50\% probability level. The hydrogens have been omitted for clarity. Selected bond distances (A) and angles (deg): Th1-O1 2.1769(18), Th1-O2 2.1771(19), O1-C1 1.366(3), O2-C9 1.363(3), O1-Th1-O2 94.29(7) Th1-O1-C1 166.22(18), Th1-O2C9 168.98(18).

Table S1. X-ray crystallographic data shown for compound 1-3.

\begin{tabular}{cccc}
\hline & $\mathbf{1}$ & $\mathbf{2}$ & $\mathbf{3}$ \\
CCDC deposit number & $166.22(18)$ & 1418299 & 1418300 \\
Empirical formula & $\mathrm{C}_{50} \mathrm{H}_{78} \mathrm{P}_{2} \mathrm{Th}$ & $\mathrm{C}_{55} \mathrm{H}_{81} \mathrm{P}_{1} \mathrm{Th}_{2}$ & $\mathrm{C}_{36} \mathrm{H}_{48} \mathrm{O}_{2} \mathrm{Th}$ \\
Formula weight $(\mathrm{g} / \mathrm{mol})$ & 973.10 & 1237.25 & 744.78 \\
Crystal habit, color & Prism, orange & Brick, red & Brick, colorless \\
Temperature $(\mathrm{K})$ & $100(2)$ & $100(2)$ & $100(2)$ \\
Space group & $P 2_{1} / \mathrm{c}$ & $P 2_{1} / \mathrm{c}$ & $P-1$ \\
Crystal system & Monoclinic & Monoclinic & Triclinic \\
Volume $\left(\AA^{3}\right)$ & $4753.5(5)$ & $5028.4(13)$ & $1608.2(6)$ \\
$a(\AA)$ & $23.0790(13)$ & $11.0279(16)$ & $9.489(2)$ \\
$b(\AA)$ & $12.1433(7)$ & $18.200(3)$ & $10.143(2)$ \\
$c\left(^{\AA} \AA\right)$ & $17.7765(10)$ & $25.124(4)$ & $18.579(4)$ \\
$\alpha\left(^{\circ}\right)$ & 90.00 & 90 & $82.039(2)$ \\
$\beta\left(^{\circ}\right)$ & $107.4180(10)$ & $94.300(2)$ & $77.460(2)$ \\
$\gamma\left({ }^{\circ}\right)$ & 90.00 & 90 & $67.375(2)$ \\
$Z$ & 4 & 4 & 2 \\
Calculated density $\left(\mathrm{Mg} / \mathrm{m}^{3}\right)$ & 1.360 & 1.634 & 1.538 \\
Absorption coefficient $\left(\mathrm{mm}^{-1}\right)$ & 3.235 & 5.973 & 4.665 \\
Final R indices $[\mathrm{I}>2 \sigma(\mathrm{I})]$ & $\mathrm{R}=0.0198$ & $\mathrm{R}=0.0207$ & $\mathrm{R}=0.0204$ \\
& $\mathrm{R}_{\mathrm{W}}=0.0440$ & $\mathrm{R}_{\mathrm{W}}=0.0431$ & $\mathrm{R}_{\mathrm{W}}=0.0423$ \\
\hline
\end{tabular}




\section{Optimized xyz coordinates}

$\left(\mathrm{C}_{5} \mathrm{Me}_{5}\right)_{2} \mathrm{Th}\left(\mathrm{CH}_{3}\right)_{2}$

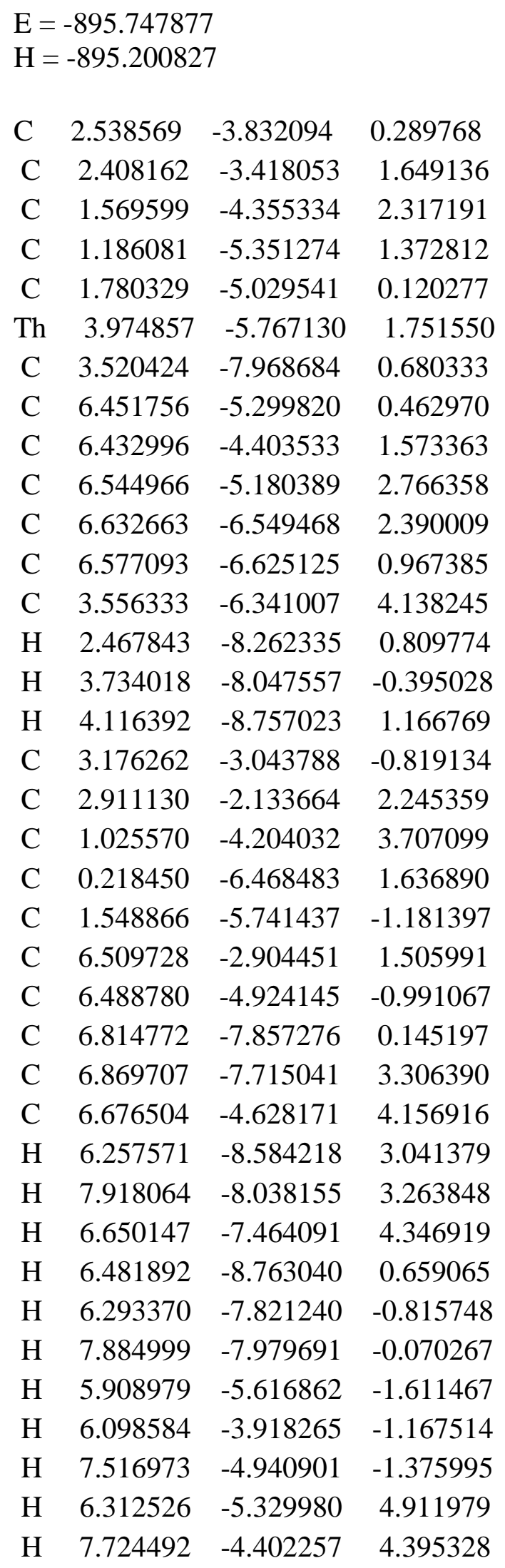




$\begin{array}{lrrr}\mathrm{H} & 6.114508 & -3.696917 & 4.283861 \\ \mathrm{H} & 5.989765 & -2.421264 & 2.339007 \\ \mathrm{H} & 7.553947 & -2.566831 & 1.546273 \\ \mathrm{H} & 6.085679 & -2.509061 & 0.578272 \\ \mathrm{H} & 3.564890 & -3.686064 & -1.615537 \\ \mathrm{H} & 2.447628 & -2.367463 & -1.285705 \\ \mathrm{H} & 4.003194 & -2.421552 & -0.464361 \\ \mathrm{H} & 3.703901 & -1.684324 & 1.641867 \\ \mathrm{H} & 2.103585 & -1.393231 & 2.316706 \\ \mathrm{H} & 3.303350 & -2.267810 & 3.260251 \\ \mathrm{H} & 0.768427 & -5.167499 & 4.155082 \\ \mathrm{H} & 1.737795 & -3.715353 & 4.378538 \\ \mathrm{H} & 0.112795 & -3.592703 & 3.702409 \\ \mathrm{H} & 0.278696 & -7.247527 & 0.872723 \\ \mathrm{H} & 0.389893 & -6.945378 & 2.607705 \\ \mathrm{H} & -0.815011 & -6.097811 & 1.641303 \\ \mathrm{H} & 2.401103 & -5.641652 & -1.861283 \\ \mathrm{H} & 1.375667 & -6.811113 & -1.037283 \\ \mathrm{H} & 0.673774 & -5.333545 & -1.704780 \\ \mathrm{H} & 4.280956 & -7.078643 & 4.513293 \\ \mathrm{H} & 3.573280 & -5.499782 & 4.845607 \\ \mathrm{H} & 2.564603 & -6.807978 & 4.247666\end{array}$

\section{$\mathrm{PH}_{2}\left(2,4,6-{ }^{i} \operatorname{Pr}_{3} \mathrm{C}_{6} \mathrm{H}_{2}\right)$}

$\begin{array}{lrrr}\mathrm{E}=-593.040482 & & \\ \mathrm{H}= & -592.657699 & & \\ \mathrm{H} & -0.035705 & -0.005631 & -0.000157 \\ \mathrm{C} & -0.038289 & -0.068837 & 1.410683 \\ \mathrm{C} & 1.182688 & -0.092741 & 2.088459 \\ \mathrm{C} & 2.406726 & -0.084681 & 1.417597 \\ \mathrm{C} & 2.380068 & -0.086649 & 0.024646 \\ \mathrm{C} & 1.185355 & -0.066005 & -0.702380 \\ \mathrm{C} & -1.330263 & -0.093275 & 2.218300 \\ \mathrm{H} & 1.176300 & -0.122063 & 3.175225 \\ \mathrm{C} & 3.724677 & -0.103518 & 2.171582 \\ \mathrm{H} & 3.324936 & -0.109448 & -0.513790 \\ \mathrm{C} & 1.249167 & -0.094515 & -2.224590 \\ \mathrm{H} & 0.258050 & -0.380335 & -2.598096 \\ \mathrm{C} & 1.577363 & 1.294821 & -2.790494 \\ \mathrm{C} & 2.223556 & -1.149935 & -2.763639 \\ \mathrm{H} & 2.142372 & -1.213330 & -3.854068 \\ \mathrm{H} & 3.265555 & -0.906442 & -2.531140\end{array}$




$\begin{array}{lrrr}\mathrm{H} & 2.008341 & -2.138995 & -2.348048 \\ \mathrm{H} & 1.569052 & 1.283546 & -3.886111 \\ \mathrm{H} & 0.858362 & 2.047471 & -2.451532 \\ \mathrm{H} & 2.571878 & 1.618877 & -2.464060 \\ \mathrm{C} & -1.650354 & 1.297060 & 2.786754 \\ \mathrm{H} & -2.146402 & -0.371694 & 1.540187 \\ \mathrm{C} & -1.325120 & -1.152307 & 3.328060 \\ \mathrm{H} & -2.315005 & -1.214052 & 3.792815 \\ \mathrm{H} & -1.072289 & -2.140869 & 2.933037 \\ \mathrm{H} & -0.610272 & -0.914479 & 4.122791 \\ \mathrm{H} & -2.608223 & 1.292901 & 3.318742 \\ \mathrm{H} & -0.873493 & 1.612293 & 3.492306 \\ \mathrm{H} & -1.702236 & 2.052161 & 1.995888 \\ \mathrm{H} & 4.524480 & -0.063102 & 1.419671 \\ \mathrm{C} & 3.882776 & 1.124114 & 3.078798 \\ \mathrm{C} & 3.905340 & -1.404261 & 2.966529 \\ \mathrm{H} & 4.862534 & 1.120076 & 3.569085 \\ \mathrm{H} & 3.790115 & 2.053631 & 2.508663 \\ \mathrm{H} & 3.119702 & 1.138033 & 3.864804 \\ \mathrm{H} & 4.885816 & -1.427991 & 3.454977 \\ \mathrm{H} & 3.143046 & -1.500480 & 3.747641 \\ \mathrm{H} & 3.826652 & -2.280604 & 2.315812 \\ \mathrm{P} & -1.659303 & 0.035305 & -0.936902 \\ \mathrm{H} & -2.315960 & 1.029424 & -0.140801 \\ \mathrm{H} & -1.291609 & 1.009557 & -1.920867\end{array}$

\section{TS1}

$\begin{array}{lclc}\mathrm{E}=-1488.751201 & & \\ \mathrm{H}=-1487.821244 & & \\ & & & \\ \mathrm{C} & 2.440677 & -4.023037 & 0.521087 \\ \mathrm{C} & 1.864346 & -3.977019 & 1.823796 \\ \mathrm{C} & 1.191181 & -5.216561 & 2.050341 \\ \mathrm{C} & 1.335174 & -6.013660 & 0.879940 \\ \mathrm{C} & 2.119924 & -5.283338 & -0.060379 \\ \mathrm{Th} & 3.957246 & -5.928257 & 2.032891 \\ \mathrm{C} & 3.932479 & -7.425610 & 4.228308 \\ \mathrm{C} & 3.534376 & -8.239381 & 1.206443 \\ \mathrm{C} & 6.151940 & -5.192570 & 0.419347 \\ \mathrm{C} & 6.416322 & -4.628823 & 1.703877 \\ \mathrm{C} & 6.708442 & -5.704307 & 2.596159 \\ \mathrm{C} & 6.627226 & -6.922009 & 1.865281 \\ \mathrm{C} & 6.271545 & -6.611112 & 0.524563\end{array}$




\begin{tabular}{|c|c|c|c|}
\hline $\mathrm{P}$ & 3.888239 & -4.191752 & 4.826339 \\
\hline $\mathrm{H}$ & 2.634708 & -8.660339 & 1.677228 \\
\hline $\mathrm{H}$ & 3.386842 & -8.326850 & 0.120299 \\
\hline 1 & 4.355127 & -8.923154 & 1.461772 \\
\hline $\mathrm{H}$ & 3.894052 & -5.831563 & 4.415404 \\
\hline C & 3.033341 & -2.829662 & -0.170448 \\
\hline C & 1.724332 & -2.714084 & 2.625737 \\
\hline $\mathrm{C}$ & 0.301163 & -5.548583 & 3.215359 \\
\hline $\mathrm{C}$ & 0.576238 & -7.272292 & 0.582788 \\
\hline $\mathrm{C}$ & 2.384275 & -5.716288 & -1.474001 \\
\hline $\mathrm{C}$ & 6.527326 & -3.165228 & 2.028963 \\
\hline $\mathrm{C}$ & 6.069828 & -4.447895 & -0.882204 \\
\hline $\mathrm{C}$ & 6.243183 & -7.570535 & -0.627901 \\
\hline $\mathrm{C}$ & 7.036546 & -8.268940 & 2.382619 \\
\hline $\mathrm{C}$ & 7.202548 & -5.580001 & 4.008117 \\
\hline $\mathrm{H}$ & 4.522442 & -8.179237 & 3.687044 \\
\hline $\mathrm{H}$ & 4.332979 & -7.405199 & 5.247454 \\
\hline $\mathrm{H}$ & 2.907382 & -7.816751 & 4.293176 \\
\hline $\mathrm{H}$ & 2.475612 & -4.166770 & 5.032030 \\
\hline $\mathrm{C}$ & 4.404781 & -4.506255 & 6.606512 \\
\hline $\mathrm{C}$ & 3.707950 & -5.385263 & 7.473784 \\
\hline $\mathrm{C}$ & 4.170961 & -5.553700 & 8.783382 \\
\hline $\mathrm{C}$ & 5.288779 & -4.887503 & 9.277804 \\
\hline $\mathrm{C}$ & 5.936224 & -3.994504 & 8.424411 \\
\hline $\mathrm{C}$ & 5.515794 & -3.779249 & 7.109735 \\
\hline $\mathrm{C}$ & 2.412004 & -6.083949 & 7.084274 \\
\hline $\mathrm{H}$ & 3.636235 & -6.228617 & 9.447488 \\
\hline $\mathrm{C}$ & 5.763891 & -5.112677 & 10.701930 \\
\hline $\mathrm{H}$ & 6.789706 & -3.435496 & 8.798153 \\
\hline $\mathrm{C}$ & 6.204908 & -2.680528 & 6.307132 \\
\hline $\mathrm{C}$ & 2.298007 & -7.525770 & 7.593428 \\
\hline $\mathrm{C}$ & 1.209645 & -5.250776 & 7.558254 \\
\hline $\mathrm{H}$ & 2.372476 & -6.127817 & 5.993136 \\
\hline $\mathrm{H}$ & 1.409075 & -8.001481 & 7.165406 \\
\hline $\mathrm{H}$ & 3.168731 & -8.124555 & 7.310636 \\
\hline $\mathrm{H}$ & 2.192257 & -7.575219 & 8.682536 \\
\hline $\mathrm{H}$ & 0.266275 & -5.712128 & 7.244275 \\
\hline $\mathrm{H}$ & 1.200135 & -5.172559 & 8.651209 \\
\hline $\mathrm{H}$ & 1.243453 & -4.232946 & 7.156410 \\
\hline $\mathrm{C}$ & 5.664034 & -3.833420 & 11.544416 \\
\hline $\mathrm{H}$ & 5.090871 & -5.856323 & 11.149996 \\
\hline $\mathrm{C}$ & 7.184604 & -5.691710 & 10.742925 \\
\hline $\mathrm{H}$ & 7.487388 & -5.904560 & 11.774193 \\
\hline $\mathrm{H}$ & 7.250163 & -6.621106 & 10.168894 \\
\hline
\end{tabular}




\begin{tabular}{cccc} 
& & & \\
$\mathrm{H}$ & 7.911710 & -4.988540 & 10.322086 \\
$\mathrm{H}$ & 5.956882 & -4.028332 & 12.582096 \\
$\mathrm{H}$ & 6.323574 & -3.049781 & 11.155442 \\
$\mathrm{H}$ & 4.643402 & -3.438486 & 11.545897 \\
$\mathrm{H}$ & 6.043832 & -2.893660 & 5.245329 \\
$\mathrm{C}$ & 5.529042 & -1.329870 & 6.589806 \\
$\mathrm{C}$ & 7.717641 & -2.586016 & 6.534278 \\
$\mathrm{H}$ & 5.993021 & -0.531106 & 5.999862 \\
$\mathrm{H}$ & 4.464393 & -1.364754 & 6.338806 \\
$\mathrm{H}$ & 5.617939 & -1.066053 & 7.649653 \\
$\mathrm{H}$ & 8.160132 & -1.890351 & 5.813280 \\
$\mathrm{H}$ & 7.962654 & -2.205626 & 7.532000 \\
$\mathrm{H}$ & 8.209569 & -3.556490 & 6.416122 \\
$\mathrm{H}$ & 6.506840 & -9.084250 & 1.882220 \\
$\mathrm{H}$ & 8.109746 & -8.432917 & 2.216487 \\
$\mathrm{H}$ & 6.860271 & -8.366874 & 3.457496 \\
$\mathrm{H}$ & 5.959666 & -8.577850 & -0.313616 \\
$\mathrm{H}$ & 5.537704 & -7.260407 & -1.404830 \\
$\mathrm{H}$ & 7.232383 & -7.639071 & -1.100542 \\
$\mathrm{H}$ & 5.356958 & -4.897615 & -1.579830 \\
$\mathrm{H}$ & 5.788641 & -3.401883 & -0.746717 \\
$\mathrm{H}$ & 7.047786 & -4.455774 & -1.381559 \\
$\mathrm{H}$ & 8.278325 & -5.793618 & 4.059564 \\
$\mathrm{H}$ & 7.054836 & -4.569889 & 4.392403 \\
$\mathrm{H}$ & 6.703148 & -6.270467 & 4.695172 \\
$\mathrm{H}$ & 5.957203 & -2.892550 & 2.923678 \\
$\mathrm{H}$ & 7.572395 & -2.880590 & 2.205100 \\
$\mathrm{H}$ & 6.160853 & -2.542972 & 1.207948 \\
$\mathrm{H}$ & 3.534121 & -3.098688 & -1.102636 \\
$\mathrm{H}$ & 2.244593 & -2.108788 & -0.425335 \\
$\mathrm{H}$ & 3.755322 & -2.295722 & 0.456570 \\
$\mathrm{H}$ & 2.679410 & -2.213281 & 2.812377 \\
$\mathrm{H}$ & 1.082107 & -2.003859 & 2.087501 \\
$\mathrm{H}$ & 1.258265 & -2.894379 & 3.596238 \\
$\mathrm{H}$ & 0.307021 & -6.618934 & 3.444821 \\
$\mathrm{H}$ & 0.596389 & -5.019242 & 4.125540 \\
$\mathrm{H}$ & -0.740723 & -5.272641 & 3.005089 \\
$\mathrm{H}$ & 1.110533 & -7.925377 & -0.111203 \\
$\mathrm{H}$ & 0.368986 & -7.854850 & 1.484966 \\
$\mathrm{H}$ & -0.392843 & -7.028788 & 0.126193 \\
$\mathrm{H}$ & 3.117116 & -5.072853 & -1.968163 \\
$\mathrm{H}$ & 2.753408 & -6.745953 & -1.531218 \\
$\mathrm{H}$ & 1.465957 & -5.673306 & -2.073930 \\
& & & \\
\hline
\end{tabular}




\section{Int1}

$$
\begin{aligned}
& E=-1448.303085 \\
& H=-1447.417371
\end{aligned}
$$

\begin{tabular}{|c|c|c|c|}
\hline 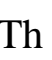 & 0.713123 & -1.340381 & -0.169302 \\
\hline $\mathrm{C}$ & -1.164933 & -1.211406 & 1.936272 \\
\hline $\mathrm{C}$ & -1.393944 & -2.496503 & 1.360737 \\
\hline $\mathrm{C}$ & -0.255859 & -3.307816 & 1.621045 \\
\hline$C$ & 0.673507 & -2.534262 & 2.381877 \\
\hline C & 0.113608 & -1.236056 & 2.567779 \\
\hline C & 3.500700 & -1.161430 & 0.023244 \\
\hline $\mathrm{C}$ & 3.156548 & -0.105297 & -0.872300 \\
\hline $\mathrm{C}$ & 2.709178 & -0.688666 & -2.093056 \\
\hline $\mathrm{C}$ & 2.785349 & -2.105941 & -1.957148 \\
\hline $\mathrm{C}$ & 3.273285 & -2.399344 & -0.651757 \\
\hline $\mathrm{C}$ & 2.616983 & -3.107158 & -3.060728 \\
\hline $\mathrm{P}$ & -0.416568 & 1.120372 & -1.212991 \\
\hline $\mathrm{C}$ & -1.701301 & 2.327299 & -0.590918 \\
\hline $\mathrm{C}$ & -1.382730 & 3.294764 & 0.402837 \\
\hline $\mathrm{C}$ & -2.360211 & 4.195010 & 0.832583 \\
\hline $\mathrm{C}$ & -3.661394 & 4.178535 & 0.333175 \\
\hline $\mathrm{C}$ & -3.953529 & 3.233195 & -0.645002 \\
\hline $\mathrm{C}$ & -3.011302 & 2.319887 & -1.137986 \\
\hline $\mathrm{C}$ & -3.506166 & 1.440118 & -2.288679 \\
\hline $\mathrm{C}$ & 4.211584 & -0.957526 & 1.330819 \\
\hline $\mathrm{C}$ & 3.435000 & 1.347237 & -0.612508 \\
\hline $\mathrm{C}$ & 2.381712 & 0.040324 & -3.364998 \\
\hline $\mathrm{C}$ & 3.657611 & -3.773266 & -0.180282 \\
\hline $\mathrm{C}$ & 1.874131 & -3.087077 & 3.093568 \\
\hline $\mathrm{C}$ & 0.685327 & -0.137484 & 3.417798 \\
\hline $\mathrm{C}$ & -2.180943 & -0.112323 & 2.052662 \\
\hline $\mathrm{C}$ & -2.685694 & -2.959244 & 0.754512 \\
\hline $\mathrm{C}$ & -0.119926 & -4.769399 & 1.305181 \\
\hline $\mathrm{C}$ & -4.715127 & 5.158763 & 0.815275 \\
\hline $\mathrm{C}$ & -5.012988 & 4.988000 & 2.311169 \\
\hline $\mathrm{C}$ & 0.017410 & 3.428270 & 0.982007 \\
\hline $\mathrm{C}$ & 0.048955 & 3.784759 & 2.473431 \\
\hline $\mathrm{C}$ & -2.809143 & 1.749252 & -3.622117 \\
\hline $\mathrm{C}$ & -4.332544 & 6.609677 & 0.491790 \\
\hline $\mathrm{C}$ & 0.835584 & 4.453937 & 0.181063 \\
\hline $\mathrm{H}$ & 0.685739 & 2.010937 & -1.383661 \\
\hline $\mathrm{H}$ & -4.957859 & 3.214723 & -1.066185 \\
\hline $\mathrm{H}$ & -2.094732 & 4.932636 & 1.585628 \\
\hline
\end{tabular}




\begin{tabular}{|c|c|c|c|}
\hline $\mathrm{H}$ & -4.557929 & 1.725507 & -2.423884 \\
\hline 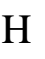 & -3.289435 & 1.191635 & -4.434761 \\
\hline $\mathrm{H}$ & -2.875832 & 2.816486 & -3.856880 \\
\hline & -1.752804 & 1.469057 & -3.589575 \\
\hline & -5.639272 & 4.931010 & 0.266636 \\
\hline & -5.813481 & 5.665874 & 2.628206 \\
\hline & -5.323029 & 3.963374 & 2.538614 \\
\hline $\mathrm{H}$ & -4.129519 & 5.211093 & 2.919699 \\
\hline $\mathrm{H}$ & -5.130376 & 7.298881 & 0.790577 \\
\hline $\mathrm{H}$ & -3.421531 & 6.906645 & 1.023176 \\
\hline $\mathrm{H}$ & -4.150195 & 6.741365 & -0.579192 \\
\hline $\mathrm{H}$ & 0.497860 & 2.447831 & 0.862813 \\
\hline $\mathrm{H}$ & 1.868668 & 4.504931 & 0.544416 \\
\hline $\mathrm{H}$ & 0.859813 & 4.206970 & -0.884505 \\
\hline $\mathrm{H}$ & 0.394654 & 5.452835 & 0.277164 \\
\hline $\mathrm{H}$ & 1.072008 & 3.702718 & 2.857204 \\
\hline $\mathrm{H}$ & -0.276736 & 4.814010 & 2.660840 \\
\hline $\mathrm{H}$ & -0.591265 & 3.122701 & 3.065259 \\
\hline $\mathrm{H}$ & -2.545110 & -3.827049 & 0.105551 \\
\hline $\mathrm{H}$ & -3.394645 & -3.250444 & 1.540884 \\
\hline $\mathrm{H}$ & -3.169063 & -2.178668 & 0.160492 \\
\hline $\mathrm{H}$ & -0.683719 & -5.046676 & 0.410559 \\
\hline $\mathrm{H}$ & 0.922269 & -5.060966 & 1.139745 \\
\hline $\mathrm{H}$ & -0.494939 & -5.386982 & 2.132260 \\
\hline $\mathrm{H}$ & 2.420061 & -3.818457 & 2.491135 \\
\hline $\mathrm{H}$ & 2.576758 & -2.304427 & 3.388917 \\
\hline $\mathrm{H}$ & 1.564722 & -3.601218 & 4.013282 \\
\hline $\mathrm{H}$ & -2.799122 & -0.256263 & 2.949465 \\
\hline $\mathbf{H}$ & -1.720026 & 0.875261 & 2.125420 \\
\hline $\mathrm{H}$ & -2.856643 & -0.081158 & 1.194667 \\
\hline $\mathrm{H}$ & 0.454063 & 0.854603 & 3.019122 \\
\hline $\mathrm{H}$ & 0.273995 & -0.175224 & 4.435257 \\
\hline $\mathrm{H}$ & 1.772853 & -0.211783 & 3.512860 \\
\hline $\mathrm{F}$ & 4.279529 & -1.880150 & 1.911068 \\
\hline $\mathrm{H}$ & 5.238611 & -0.610212 & 1.157069 \\
\hline $\mathrm{H}$ & 3.729653 & -0.202386 & 1.962116 \\
\hline $\mathrm{H}$ & 3.095849 & 1.671540 & 0.376951 \\
\hline $\mathrm{H}$ & 4.514462 & 1.544864 & -0.662309 \\
\hline $\mathrm{H}$ & 2.952949 & 1.992853 & -1.349328 \\
\hline $\mathrm{H}$ & 1.598254 & -0.466254 & -3.935955 \\
\hline $\mathrm{H}$ & 2.031990 & 1.058321 & -3.178623 \\
\hline $\mathrm{H}$ & 3.267104 & 0.106202 & -4.011101 \\
\hline H & 2.234408 & -4.064794 & -2.697214 \\
\hline $\mathrm{H}$ & 1.929345 & -2.754890 & -3.833291 \\
\hline
\end{tabular}




$\begin{array}{lrrr}\mathrm{H} & 3.580930 & -3.304554 & -3.548976 \\ \mathrm{H} & 3.907215 & -3.788310 & 0.883215 \\ \mathrm{H} & 2.866434 & -4.513023 & -0.348313 \\ \mathrm{H} & 4.542176 & -4.131847 & -0.722154 \\ \mathrm{C} & -0.387905 & -2.815255 & -1.817170 \\ \mathrm{H} & -1.481208 & -2.763060 & -1.719969 \\ \mathrm{H} & -0.117787 & -3.879864 & -1.766942 \\ \mathrm{H} & -0.156152 & -2.479099 & -2.837443 \\ \mathrm{C} & -3.522859 & -0.063050 & -1.989613 \\ \mathrm{H} & -4.029012 & -0.602268 & -2.798778 \\ \mathrm{H} & -2.510943 & -0.469461 & -1.908489 \\ \mathrm{H} & -4.063951 & -0.270826 & -1.060018\end{array}$
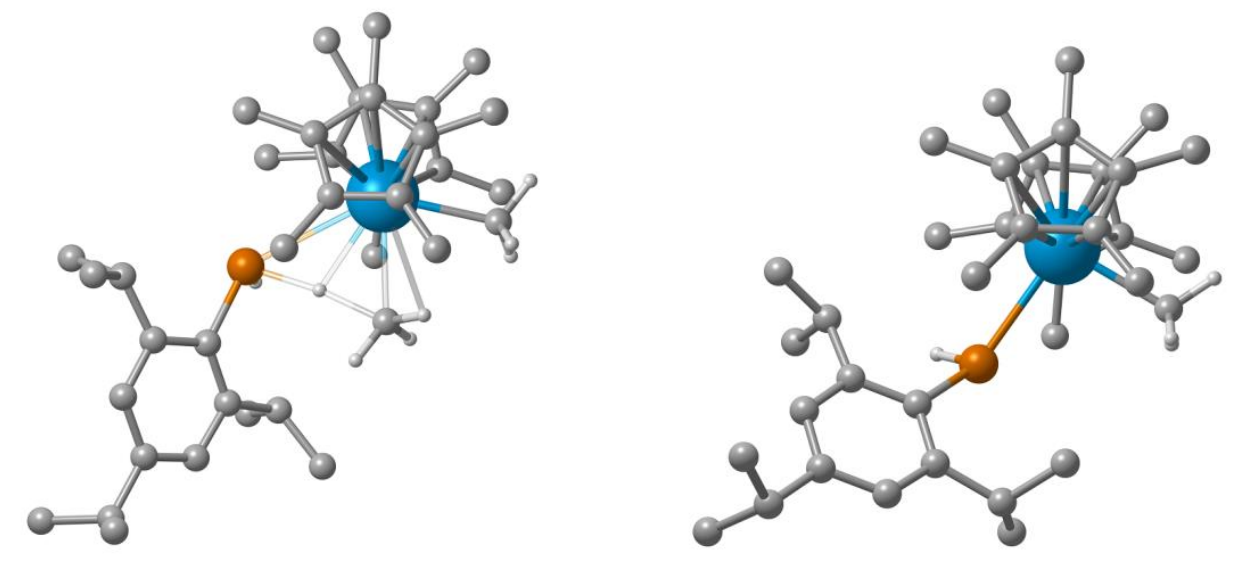

Figure S6. Optimized structures of TS1 (left) and Int1 (right). The majority of hydrogen atoms have been omitted for clarity.

\section{TS2}

$\begin{array}{lrrr}\mathrm{E}=-1448.249652 & \\ \mathrm{H}=-1447.368205 & & \\ & & & \\ \text { Th } & 0.534304 & -0.844007 & -0.226878 \\ \mathrm{C} & -0.542002 & -0.186964 & 2.324570 \\ \mathrm{C} & -1.406868 & -1.194769 & 1.810111 \\ \mathrm{C} & -0.693048 & -2.431358 & 1.818065 \\ \mathrm{C} & 0.611248 & -2.184392 & 2.335385 \\ \mathrm{C} & 0.714661 & -0.792842 & 2.623978 \\ \mathrm{C} & 3.210129 & -1.665016 & -0.395538 \\ \mathrm{C} & 3.209769 & -0.389814 & -1.036433 \\ \mathrm{C} & 2.528356 & -0.520968 & -2.279416 \\ \mathrm{C} & 2.077556 & -1.865394 & -2.396669\end{array}$




\begin{tabular}{|c|c|c|c|}
\hline & & & \\
\hline & & & \\
\hline & & & \\
\hline & & & \\
\hline & & & \\
\hline & & & \\
\hline & & & \\
\hline & & & \\
\hline & & & \\
\hline & & & \\
\hline & & & \\
\hline & & & \\
\hline & & & \\
\hline & & & \\
\hline & & & \\
\hline & & & \\
\hline & & & \\
\hline & & & \\
\hline & & & \\
\hline & & & \\
\hline & & & \\
\hline & & & \\
\hline & & & \\
\hline & & & \\
\hline & & & \\
\hline & & & \\
\hline & & & \\
\hline & & & \\
\hline & -2 & & \\
\hline & -2.9 & & \\
\hline & & & \\
\hline & -3.0 & & \\
\hline & -1.309672 & & \\
\hline & -5.862662 & & \\
\hline & -6.63 & & \\
\hline & -5.35 & & \\
\hline & -4.989543 & & \\
\hline & -6.656059 & & \\
\hline & -5.0 & 146 & \\
\hline & -5.3 & & \\
\hline & & & \\
\hline & & & \\
\hline & & & \\
\hline & 0.491930 & 6.267256 & -0.12395 \\
\hline
\end{tabular}




$\begin{array}{crrc}\mathrm{H} & 0.125244 & 5.868880 & 2.975626 \\ \mathrm{H} & -1.456510 & 6.190445 & 2.275068 \\ \mathrm{H} & -1.209609 & 4.741930 & 3.267701 \\ \mathrm{H} & -3.255391 & -1.780484 & 0.841896 \\ \mathrm{H} & -3.456392 & -1.101015 & 2.452824 \\ \mathrm{H} & -3.095016 & -0.039484 & 1.090939 \\ \mathrm{H} & -2.160407 & -3.778021 & 0.963046 \\ \mathrm{H} & -0.559328 & -4.493781 & 1.150435 \\ \mathrm{H} & -1.581216 & -4.237627 & 2.561569 \\ \mathrm{H} & 1.694162 & -4.051326 & 2.032896 \\ \mathrm{H} & 2.543067 & -2.866465 & 3.032361 \\ \mathrm{H} & 1.167715 & -3.750285 & 3.681787 \\ \mathrm{H} & -1.259831 & 1.133935 & 3.838237 \\ \mathrm{H} & -0.153466 & 1.906210 & 2.698140 \\ \mathrm{H} & -1.809697 & 1.562869 & 2.215026 \\ \mathrm{H} & 2.083631 & 0.871705 & 2.862877 \\ \mathrm{H} & 1.593342 & 0.108864 & 4.371331 \\ \mathrm{H} & 2.761297 & -0.693367 & 3.325074 \\ \mathrm{H} & 3.820977 & -2.991247 & 1.203492 \\ \mathrm{H} & 5.134769 & -2.124969 & 0.415551 \\ \mathrm{H} & 4.094518 & -1.281136 & 1.554545 \\ \mathrm{H} & 4.081121 & 0.864018 & 0.499819 \\ \mathrm{H} & 4.998071 & 0.804527 & -1.004325 \\ \mathrm{H} & 3.503711 & 1.745527 & -0.918973 \\ \mathrm{H} & 1.728140 & 0.307800 & -4.107543 \\ \mathrm{H} & 2.285555 & 1.519846 & -2.946836 \\ \mathrm{H} & 3.456213 & 0.567538 & -3.872382 \\ \mathrm{H} & 0.919009 & -3.395989 & -3.394151 \\ \mathrm{H} & 0.764653 & -1.794593 & -4.119583 \\ \mathrm{H} & 2.238107 & -2.732954 & -4.354728 \\ \mathrm{H} & 2.456199 & -4.354933 & 0.004882 \\ \mathrm{H} & 1.484910 & -4.483522 & -1.462518 \\ \mathrm{H} & 3.239895 & -4.570380 & -1.556393 \\ \mathrm{C} & -1.178365 & -2.501879 & -1.489407 \\ \mathrm{H} & -2.151350 & -2.550856 & -0.991987 \\ \mathrm{H} & -0.543724 & -3.286427 & -1.053386 \\ \mathrm{H} & -1.325630 & -2.780538 & -2.538204 \\ \mathrm{C} & -0.836842 & 0.187571 & -2.352993 \\ \mathrm{H} & -1.021128 & -0.332227 & -3.307529 \\ \mathrm{H} & 0.064977 & 0.773435 & -2.561991 \\ \mathrm{H} & -0.967488 & -1.180190 & -1.801061\end{array}$

\section{Int2}




$$
\begin{aligned}
& E=-1407.790161 \\
& H=-1406.955820
\end{aligned}
$$

$\begin{array}{cccc}\text { Th } & 1.006163 & -0.548620 & 0.065973 \\ \text { C } & 0.217787 & 0.262488 & 2.662100 \\ \text { C } & -0.872585 & -0.538125 & 2.209826 \\ \text { C } & -0.421527 & -1.885085 & 2.127325 \\ \text { C } & 0.937798 & -1.927110 & 2.564280 \\ \text { C } & 1.333271 & -0.597827 & 2.887347 \\ \text { C } & 3.603565 & -1.515542 & -0.370852 \\ \text { C } & 3.544500 & -0.330533 & -1.160962 \\ \text { C } & 2.687469 & -0.579929 & -2.272807 \\ \text { C } & 2.180477 & -1.902073 & -2.149189 \\ \text { C } & 2.743880 & -2.483423 & -0.971414 \\ \text { C } & 1.371219 & -2.647890 & -3.169741 \\ \text { P } & 0.735152 & 2.200824 & -0.851871 \\ \text { C } & -1.083517 & 2.489263 & -0.496404 \\ \text { C } & -1.532275 & 3.630880 & 0.232939 \\ \text { C } & -2.902511 & 3.832225 & 0.422693 \\ \text { C } & -3.869470 & 2.976854 & -0.104855 \\ \text { C } & -3.415500 & 1.896787 & -0.855372 \\ \text { C } & -2.058207 & 1.626171 & -1.069641 \\ \text { C } & -1.770029 & 0.426598 & -1.979905 \\ \text { C } & -1.049072 & -0.775543 & -1.312829 \\ \text { C } & 4.564252 & -1.749346 & 0.758902 \\ \text { C } & 4.368511 & 0.909867 & -0.962821 \\ \text { C } & 2.535919 & 0.327074 & -3.456550 \\ \text { C } & 2.614125 & -3.932640 & -0.595336 \\ \text { C } & 1.692377 & -3.187641 & 2.874483 \\ \text { C } & 2.630591 & -0.172251 & 3.515010 \\ \text { C } & 0.138691 & 1.707979 & 3.057794 \\ \text { C } & -2.287343 & -0.067958 & 2.049307 \\ \text { C } & -1.265590 & -3.084658 & 1.802793 \\ \text { C } & -5.353198 & 3.221604 & 0.101893 \\ \text { C } & -5.740877 & 3.196013 & 1.586548 \\ \text { C } & -0.571876 & 4.704369 & 0.737861 \\ \text { C } & -1.093004 & 5.531328 & 1.917851 \\ \text { C } & -1.127066 & 0.865034 & -3.303404 \\ \text { C } & -5.810744 & 4.527495 & -0.563178 \\ \text { C } & -0.162607 & 5.630667 & -0.419072 \\ \text { H } & 1.307274 & 2.842173 & 0.298690 \\ & -4.149441 & 1.224116 & -1.297675 \\ \text { H } & -2.768782 & 0.065695 & -2.267968\end{array}$




\begin{tabular}{|c|c|c|c|}
\hline $\mathrm{H}$ & -1.037049 & 0.007800 & -3.9 \\
\hline $\mathrm{H}$ & -1.747952 & 1.620208 & -3.797174 \\
\hline $\mathrm{H}$ & -0.132533 & 1.297488 & -3.167012 \\
\hline $\mathrm{H}$ & -5.884720 & 2.396515 & -0.392047 \\
\hline & -6.823357 & 3.316190 & 1.708349 \\
\hline & -5.447087 & 2.252124 & 2.056123 \\
\hline & -5.254080 & 4.007791 & 2.138484 \\
\hline & -6.892790 & 4.663339 & -0.453913 \\
\hline & -5.319296 & 5.395152 & -0.109123 \\
\hline & -5.571359 & 4.531833 & -1.631011 \\
\hline & 0.335243 & 4.203059 & 1.092192 \\
\hline & 0.576123 & 6.368764 & -0.085659 \\
\hline & 0.272571 & 5.062693 & -1.246693 \\
\hline & -1.036615 & 6.171522 & -0.799346 \\
\hline & -0.286338 & 6.159191 & 2.311075 \\
\hline & -1.905752 & 6.206016 & 1.626164 \\
\hline & -1.455947 & 4.898004 & 2.734125 \\
\hline $\mathrm{H}$ & -2.842924 & -0.662719 & 1.319171 \\
\hline $\mathrm{H}$ & -2.821601 & -0.148107 & 3.005679 \\
\hline $\mathrm{H}$ & -2.342751 & 0.973350 & 1.725111 \\
\hline $\mathrm{H}$ & -2.126092 & -2.821294 & 1.181646 \\
\hline $\mathrm{H}$ & -0.701975 & -3.859875 & 1.271712 \\
\hline $\mathrm{H}$ & -1.658620 & -3.550122 & 2.716525 \\
\hline $\mathrm{H}$ & 1.493801 & -3.983722 & 2.151009 \\
\hline $\mathrm{H}$ & 2.773634 & -3.031694 & 2.910412 \\
\hline $\mathrm{H}$ & 1.395488 & -3.574505 & 3.858501 \\
\hline $\mathrm{H}$ & -0.182336 & 1.805210 & 4.104078 \\
\hline $\mathrm{H}$ & 1.105899 & 2.212365 & 2.971819 \\
\hline $\mathrm{H}$ & -0.578043 & 2.256149 & 2.441389 \\
\hline $\mathrm{H}$ & 3.033478 & 0.745611 & 3.069730 \\
\hline $\mathrm{H}$ & 2.496720 & 0.034548 & 4.584739 \\
\hline $\mathrm{H}$ & 3.399710 & -0.943892 & 3.431297 \\
\hline $\mathrm{H}$ & 4.238063 & -2.555162 & 1.420959 \\
\hline $\mathrm{H}$ & 5.551406 & -2.032531 & 0.370643 \\
\hline $\mathrm{H}$ & 4.710937 & -0.855337 & 1.372437 \\
\hline $\mathrm{H}$ & 4.634746 & 1.068239 & 0.087338 \\
\hline $\mathrm{H}$ & 5.311619 & 0.848940 & -1.522555 \\
\hline $\mathrm{H}$ & 3.844318 & 1.804945 & -1.311996 \\
\hline $\mathrm{H}$ & 1.677657 & 0.053797 & -4.073920 \\
\hline $\mathrm{H}$ & 2.409462 & 1.372399 & -3.159848 \\
\hline 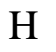 & 3.428708 & 0.265720 & -4.093118 \\
\hline $\mathrm{H}$ & 0.687737 & -3.368684 & -2.709118 \\
\hline $\mathrm{H}$ & 0.769293 & -1.974302 & -3.784436 \\
\hline $\mathrm{H}$ & 2.024739 & -3.214403 & -3.846750 \\
\hline
\end{tabular}




$\begin{array}{cccc}\mathrm{H} & 3.014505 & -4.134396 & 0.400375 \\ \mathrm{H} & 1.575319 & -4.283875 & -0.613114 \\ \mathrm{H} & 3.168971 & -4.565496 & -1.300144 \\ \mathrm{H} & -0.915984 & -1.545749 & -2.089426 \\ \mathrm{H} & -1.767372 & -1.226345 & -0.605466\end{array}$
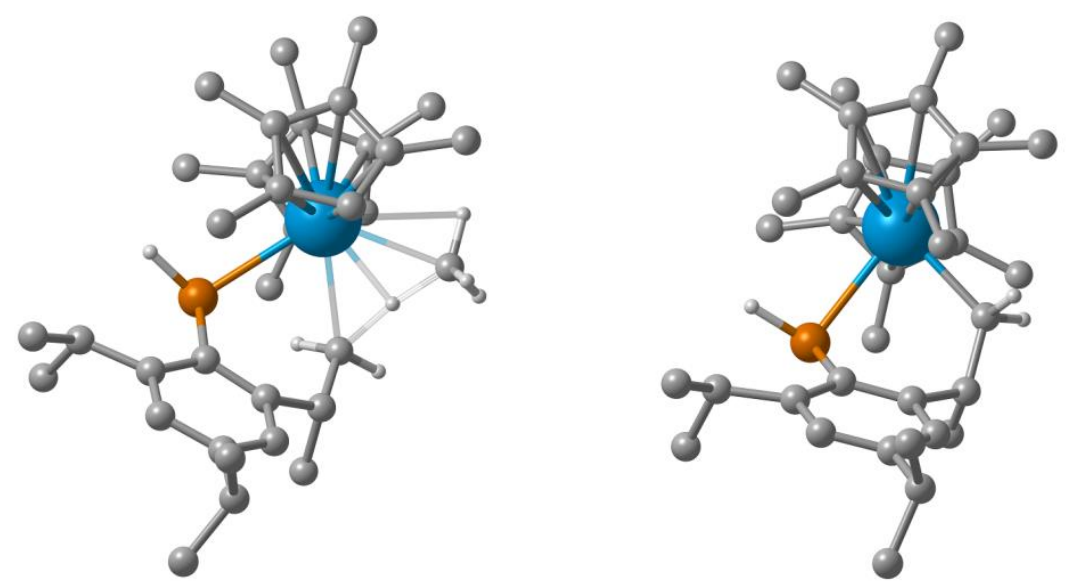

Figure S7. Optimized structures of TS2 (left) and Int2 (right). Some methyl carbons and the majority of hydrogen atoms have been omitted for clarity. 


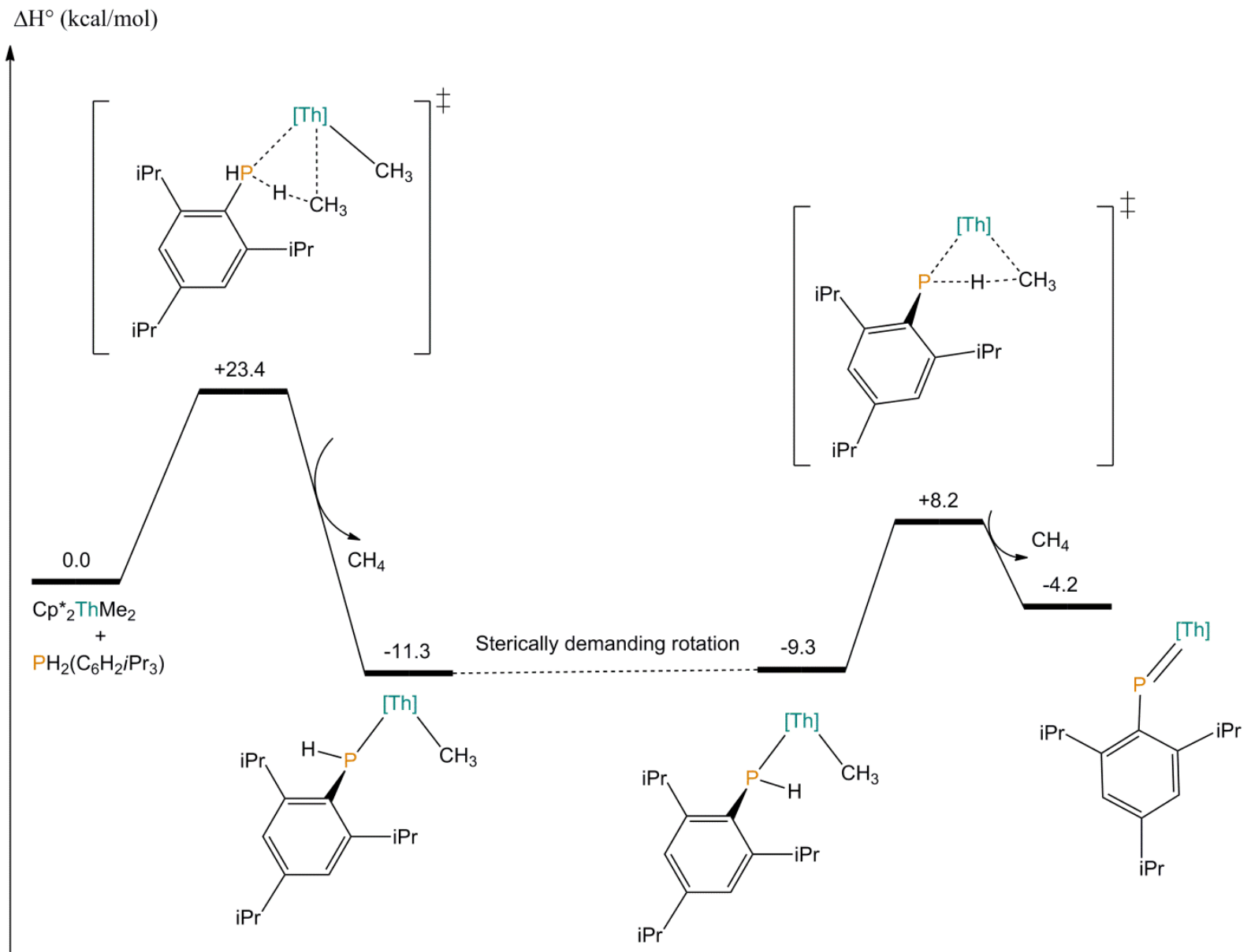

Figure S8. Energy profile of the formation of $[\mathrm{Th}]=\mathrm{P}\left(\mathrm{C}_{6} \mathrm{H}_{2}{ }^{i} \mathrm{Pr}_{3}\right)$.

Transition state $\mathrm{P}-\mathrm{H}$ activation, to form $[\mathrm{Th}]=\mathbf{P}\left(\mathrm{C}_{6} \mathrm{H}_{2}{ }^{i} \mathrm{Pr}_{3}\right)$ and $\mathrm{CH}_{4}$
$\mathrm{E}=-1448.270543$
$\mathrm{H}=-1447.386257$
$\begin{array}{llll}\text { C } & -0.088456 & 0.688755 & 1.158146\end{array}$
C $\quad-1.149443 \quad 0.966049 \quad 2.066293$
C $\quad-0.781583 \quad 2.096618 \quad 2.856212$
C $\quad 0.516342 \quad 2.514030 \quad 2.441834$
C $\quad 0.948008 \quad 1.634398 \quad 1.399852$
$\begin{array}{llll}\text { Th } & 1.008267 & 0.015938 & 3.665471\end{array}$
C $\quad 2.484205 \quad 1.266812 \quad 5.737843$
$\begin{array}{llll}\text { C } & 2.805397 & -0.121782 & 5.813707\end{array}$
C $\quad 1.653528 \quad-0.814739 \quad 6.276185$ 


$\begin{array}{lrrr}\text { C } & 0.617104 & 0.144843 & 6.490975 \\ \text { C } & 1.138203 & 1.434629 & 6.180016 \\ \text { C } & 4.164958 & -0.718999 & 5.595250 \\ \text { C } & 1.599186 & -2.276737 & 6.613740 \\ \text { C } & -0.718462 & -0.129817 & 7.122721 \\ \text { C } & 0.483519 & 2.740869 & 6.521218 \\ \text { C } & 3.474001 & 2.362434 & 5.457358 \\ \text { C } & -2.516805 & 0.347140 & 2.034961 \\ \text { C } & -1.721030 & 2.826498 & 3.773241 \\ \text { C } & 1.225011 & 3.780907 & 2.827039 \\ \text { C } & 2.212599 & 1.794883 & 0.605256 \\ \text { C } & -0.134940 & -0.320655 & 0.048770 \\ \text { C } & -0.831572 & -1.882063 & 3.898087 \\ \text { P } & 2.202522 & -1.990221 & 2.568955 \\ \text { C } & 2.586850 & -3.499148 & 1.564335 \\ \text { C } & 2.058036 & -4.774340 & 1.880039 \\ \text { C } & 2.388335 & -5.869359 & 1.076168 \\ \text { C } & 3.245164 & -5.769869 & -0.017356 \\ \text { C } & 3.785782 & -4.512708 & -0.293013 \\ \text { C } & 3.478242 & -3.381749 & 0.464143 \\ \text { C } & 1.134031 & -5.009206 & 3.063624 \\ \text { C } & -0.286114 & -5.368816 & 2.606312 \\ \text { C } & 4.152601 & -2.065464 & 0.110115 \\ \text { C } & 5.613267 & -2.057075 & 0.583510 \\ \text { C } & 3.571279 & -6.983217 & -0.869159 \\ \text { C } & 5.071087 & -7.308898 & -0.857935 \\ \text { C } & 4.053007 & -1.715500 & -1.379111 \\ \text { C } & 3.054146 & -6.822608 & -2.305651 \\ \text { C } & 1.686899 & -6.066915 & 4.028223 \\ \text { H } & 0.464833 & -2.050434 & 3.214817 \\ \text { H } & -1.487735 & -1.108290 & 4.330152 \\ \text { H } & -1.407511 & -2.335733 & 3.087054 \\ \text { H } & -0.687324 & -2.638566 & 4.674827 \\ \text { H } & 1.963459 & -6.842701 & 1.316881 \\ \text { H } & 4.473175 & -4.405567 & -1.129387 \\ \text { H } & 1.082792 & -4.065035 & 3.617715 \\ \text { H } & -0.962333 & -5.460482 & 3.464091 \\ \text { H } & -0.689629 & -4.607215 & 1.931867 \\ \text { H } & -0.299215 & -6.324533 & 2.069755 \\ \text { H } & 1.040288 & -6.158871 & 4.908405 \\ \text { H } & 1.744372 & -7.055492 & 3.559443 \\ \text { H } & 2.692239 & -5.802049 & 4.369997 \\ \text { H } & 3.045244 & -7.838438 & -0.422908 \\ \text { H } & 3.242831 & -7.728931 & -2.892320\end{array}$




$\begin{array}{cccc}\mathrm{H} & 1.977873 & -6.624649 & -2.318298 \\ \mathrm{H} & 3.551185 & -5.988395 & -2.813208 \\ \mathrm{H} & 5.275725 & -8.221640 & -1.428898 \\ \mathrm{H} & 5.656316 & -6.499357 & -1.308107 \\ \mathrm{H} & 5.436925 & -7.455160 & 0.163059 \\ \mathrm{H} & 3.623977 & -1.283339 & 0.669992 \\ \mathrm{H} & 6.084542 & -1.087738 & 0.382409 \\ \mathrm{H} & 5.678682 & -2.251231 & 1.658596 \\ \mathrm{H} & 6.197903 & -2.828124 & 0.068294 \\ \mathrm{H} & 4.458564 & -0.713933 & -1.562888 \\ \mathrm{H} & 4.621721 & -2.411423 & -2.005990 \\ \mathrm{H} & 3.014345 & -1.732058 & -1.724452 \\ \mathrm{H} & -2.991250 & 0.329384 & 3.021137 \\ \mathrm{H} & -3.180679 & 0.917771 & 1.371702 \\ \mathrm{H} & -2.494180 & -0.679339 & 1.660478 \\ \mathrm{H} & -2.216124 & 2.161416 & 4.490570 \\ \mathrm{H} & -1.213833 & 3.606406 & 4.344201 \\ \mathrm{H} & -2.518026 & 3.314242 & 3.196976 \\ \mathrm{H} & 0.875262 & 4.180577 & 3.782358 \\ \mathrm{H} & 2.309241 & 3.648908 & 2.899811 \\ \mathrm{H} & 1.053564 & 4.560007 & 2.072393 \\ \mathrm{H} & -0.666547 & 0.086343 & -0.822263 \\ \mathrm{H} & 0.866124 & -0.612593 & -0.273873 \\ \mathrm{H} & -0.650006 & -1.237316 & 0.349416 \\ \mathrm{H} & 2.483757 & 0.871001 & 0.090280 \\ \mathrm{H} & 2.100484 & 2.577990 & -0.156890 \\ \mathrm{H} & 3.065893 & 2.082173 & 1.229923 \\ \mathrm{H} & 2.982530 & 3.308322 & 5.217070 \\ \mathrm{H} & 4.111793 & 2.544396 & 6.332458 \\ \mathrm{H} & 4.146900 & 2.116683 & 4.627883 \\ \mathrm{H} & 4.732124 & -0.181609 & 4.829253 \\ \mathrm{H} & 4.755343 & -0.683792 & 6.521365 \\ \mathrm{H} & 4.098435 & -1.760381 & 5.273140 \\ \mathrm{H} & 0.570587 & -2.636812 & 6.703943 \\ \mathrm{H} & 2.099334 & -2.886552 & 5.855086 \\ \mathrm{H} & 2.091685 & -2.471924 & 7.575440 \\ \mathrm{H} & -1.473073 & 0.607264 & 6.829416 \\ \mathrm{H} & -1.105686 & -1.118026 & 6.857878 \\ \mathrm{H} & -0.649510 & -0.095372 & 8.218009 \\ \mathrm{H} & 0.812076 & 3.555478 & 5.870056 \\ \mathrm{H} & -0.606690 & 2.689148 & 6.463687 \\ \mathrm{H} & 0.734825 & 3.036140 & 7.548887\end{array}$




$\begin{array}{cccc}\text { [Th }]=\mathrm{P}_{(}\left(\mathrm{C}_{6} \mathrm{H}_{2} \mathrm{PPr}\right. & & \\ & & \\ \mathrm{E}= & -1407.785916 & & \\ \mathrm{H}= & -1406.946723 & & \\ & & & \\ \mathrm{C} & -1.821040 & 1.951093 & -1.961592 \\ \mathrm{C} & -2.770108 & 2.061432 & -0.911518 \\ \mathrm{C} & -2.472795 & 2.900170 & 0.191528 \\ \mathrm{C} & -1.260881 & 3.595399 & 0.215978 \\ \mathrm{C} & -0.319802 & 3.502757 & -0.807987 \\ \mathrm{C} & -0.628226 & 2.671274 & -1.886749 \\ \mathrm{P} & -4.368039 & 1.138299 & -0.986459 \\ \mathrm{Th} & -6.512216 & -0.206357 & -1.013075 \\ \mathrm{C} & -9.169614 & 0.026979 & -2.053826 \\ \mathrm{C} & -8.283214 & 0.294416 & -3.140534 \\ \mathrm{C} & -7.628337 & 1.538216 & -2.889428 \\ \mathrm{C} & -8.076470 & 2.020335 & -1.627360 \\ \mathrm{C} & -9.020301 & 1.080439 & -1.105772 \\ \mathrm{C} & -8.195479 & -0.500437 & -4.414225 \\ \mathrm{C} & -6.747627 & 2.281136 & -3.851275 \\ \mathrm{C} & -7.738481 & 3.355364 & -1.027312 \\ \mathrm{C} & -9.835527 & 1.272175 & 0.143519 \\ \mathrm{C} & -10.232522 & -1.031721 & -2.041943 \\ \mathrm{C} & -3.428595 & 3.070169 & 1.362162 \\ \mathrm{C} & -3.892286 & 4.524127 & 1.517365 \\ \mathrm{C} & 0.985701 & 4.274760 & -0.743597 \\ \mathrm{C} & 2.200648 & 3.337682 & -0.693335 \\ \mathrm{C} & -2.069283 & 1.082557 & -3.185540 \\ \mathrm{C} & -0.963685 & 0.041393 & -3.398588 \\ \mathrm{C} & -5.150122 & -2.137157 & 0.502796 \\ \mathrm{C} & -6.395154 & -1.950362 & 1.167307 \\ \mathrm{C} & -7.430362 & -2.513776 & 0.353718 \\ \mathrm{C} & -6.826806 & -3.004177 & -0.837149 \\ \mathrm{C} & -5.418391 & -2.752459 & -0.752573 \\ \mathrm{C} & -3.790842 & -1.872902 & 1.077312 \\ \mathrm{C} & -6.585214 & -1.405423 & 2.556195 \\ \mathrm{C} & -8.847052 & -2.724739 & 0.804702 \\ \mathrm{C} & -7.487515 & -3.815582 & -1.916057 \\ \mathrm{C} & -4.384916 & -3.206251 & -1.745835 \\ \mathrm{C} & -2.825843 & 2.535309 & 2.667630 \\ \mathrm{C} & -2.278956 & 1.937443 & -4.442414 \\ \mathrm{C} & 1.115441 & 5.280480 & -1.895886 \\ \mathrm{H} & -1.040547 & 4.237016 & 1.068369 \\ \mathrm{H} & 0.085726 & 2.579231 & -2.703517\end{array}$




\begin{tabular}{|c|c|c|c|}
\hline $\mathrm{H}$ & -4.315224 & 2.463873 & 1.135430 \\
\hline $\mathrm{H}$ & -3.548995 & 2.607380 & 3.488901 \\
\hline $\mathrm{H}$ & -2.531009 & 1.486366 & 2.562371 \\
\hline $\mathrm{H}$ & -1.934858 & 3.101781 & 2.961950 \\
\hline $\mathrm{H}$ & -4.623714 & 4.613834 & 2.329344 \\
\hline $\mathrm{H}$ & -3.056097 & 5.192512 & 1.752226 \\
\hline $\mathrm{H}$ & -4.359088 & 4.887606 & 0.596511 \\
\hline $\mathrm{H}$ & 0.972889 & 4.848112 & 0.193745 \\
\hline $\mathrm{H}$ & 3.130786 & 3.908078 & -0.588403 \\
\hline $\mathrm{H}$ & 2.128635 & 2.642275 & 0.148693 \\
\hline $\mathrm{H}$ & 2.277968 & 2.741986 & -1.609758 \\
\hline $\mathrm{H}$ & 2.036258 & 5.867331 & -1.800636 \\
\hline $\mathrm{H}$ & 1.146363 & 4.770199 & -2.865021 \\
\hline $\mathrm{H}$ & 0.268323 & 5.973165 & -1.912747 \\
\hline $\mathrm{H}$ & -3.003693 & 0.537360 & -3.000508 \\
\hline $\mathrm{H}$ & -2.509430 & 1.307424 & -5.310003 \\
\hline $\mathrm{H}$ & -3.105191 & 2.641307 & -4.302219 \\
\hline $\mathrm{H}$ & -1.382136 & 2.519736 & -4.683746 \\
\hline $\mathrm{H}$ & -1.210547 & -0.615692 & -4.240787 \\
\hline $\mathrm{H}$ & 0.001634 & 0.509516 & -3.622425 \\
\hline $\mathrm{H}$ & -0.830916 & -0.580950 & -2.507873 \\
\hline $\mathrm{H}$ & -7.537917 & -0.875184 & 2.668999 \\
\hline $\mathrm{H}$ & -6.588038 & -2.212596 & 3.300763 \\
\hline $\mathrm{H}$ & -5.785019 & -0.714090 & 2.836153 \\
\hline $\mathrm{H}$ & -9.287358 & -1.828795 & 1.257028 \\
\hline $\mathrm{H}$ & -9.497065 & -3.031654 & -0.017192 \\
\hline $\mathrm{H}$ & -8.893730 & -3.514214 & 1.566138 \\
\hline I & -8.575242 & -3.710362 & -1.903128 \\
\hline $\mathrm{H}$ & -7.142013 & -3.546350 & -2.920735 \\
\hline $\mathrm{H}$ & -7.266728 & -4.883163 & -1.787557 \\
\hline $\mathrm{H}$ & -3.418413 & -2.765753 & 1.598760 \\
\hline $\mathrm{H}$ & -3.070521 & -1.601344 & 0.303003 \\
\hline $\mathrm{H}$ & -3.806731 & -1.048702 & 1.793586 \\
\hline $\mathrm{H}$ & -3.512736 & -2.546892 & -1.746978 \\
\hline $\mathrm{H}$ & -4.033047 & -4.220731 & -1.514699 \\
\hline $\mathrm{H}$ & -4.776918 & -3.236417 & -2.768275 \\
\hline $\mathrm{H}$ & -8.401939 & -1.563700 & -4.254658 \\
\hline $\mathrm{H}$ & -8.926325 & -0.143056 & -5.151663 \\
\hline $\mathrm{H}$ & -7.210779 & -0.418450 & -4.885953 \\
\hline $\mathrm{H}$ & -6.168616 & 1.605204 & -4.486750 \\
\hline 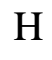 & -7.350906 & 2.916382 & -4.514472 \\
\hline 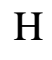 & -6.032176 & 2.916446 & -3.325522 \\
\hline $\mathrm{H}$ & -7.826404 & 3.351390 & 0.063568 \\
\hline $\mathrm{H}$ & -6.715751 & 3.654248 & -1.268538 \\
\hline
\end{tabular}




$\begin{array}{cccc}\mathrm{H} & -8.419132 & 4.132807 & -1.400417 \\ \mathrm{H} & -10.211822 & 0.325244 & 0.542346 \\ \mathrm{H} & -9.267401 & 1.764610 & 0.940411 \\ \mathrm{H} & -10.710258 & 1.906227 & -0.051543 \\ \mathrm{H} & -9.892054 & -1.976125 & -2.476868 \\ \mathrm{H} & -10.596700 & -1.241263 & -1.033450 \\ \mathrm{H} & -11.099648 & -0.707420 & -2.632830\end{array}$

\section{TS3}

$$
\begin{aligned}
& E=-2303.486912 \\
& H=-2302.106796
\end{aligned}
$$

$\begin{array}{crrc}\text { Th } & 1.485452 & 16.172697 & 9.850242 \\ \text { C } & -0.794606 & 14.684569 & 8.983301 \\ \text { C } & 0.082953 & 14.677088 & 7.859382 \\ \text { C } & 1.227092 & 13.899030 & 8.199848 \\ \text { C } & 1.052263 & 13.411625 & 9.528598 \\ \text { C } & -0.193993 & 13.909381 & 10.016787 \\ \text { C } & 3.811363 & 16.907446 & 11.283745 \\ \text { C } & 2.799099 & 17.776673 & 11.782741 \\ \text { C } & 1.875183 & 16.993498 & 12.531349 \\ \text { C } & 2.324852 & 15.638660 & 12.505773 \\ \text { C } & 3.518124 & 15.585006 & 11.726452 \\ \text { P } & 2.846135 & 17.192344 & 7.427044 \\ \text { C } & 2.453113 & 18.993221 & 7.803266 \\ \text { C } & 1.206268 & 19.335118 & 8.387060 \\ \text { C } & 0.956682 & 20.647955 & 8.791796 \\ \text { C } & 1.888728 & 21.669152 & 8.601961 \\ \text { C } & 3.062897 & 21.340829 & 7.922758 \\ \text { C } & 3.353682 & 20.043560 & 7.486980 \\ \text { C } & 1.631338 & 23.086192 & 9.083289 \\ \text { C } & 0.365519 & 23.689556 & 8.459725 \\ \text { C } & 1.575512 & 23.163822 & 10.615364 \\ \text { C } & 0.068589 & 18.320453 & 8.513161 \\ \text { C } & -1.151813 & 18.777428 & 7.693560 \\ \text { C } & -0.265964 & 17.908630 & 9.957687\end{array}$




\begin{tabular}{|c|c|c|c|}
\hline $\mathrm{C}$ & 2.810806 & 2773 & 11. \\
\hline $\mathrm{C}$ & 5.094175 & 17.333189 & 10.634087 \\
\hline C & 4.451694 & 14.417141 & 11.578610 \\
\hline $\mathrm{C}$ & 1.786158 & 14.549319 & 13.387379 \\
\hline $\mathrm{C}$ & 0.729313 & 17.505109 & 13.355903 \\
\hline $\mathrm{C}$ & -0.890198 & 13.510308 & 11.286384 \\
\hline C & -2.209391 & 15.182054 & 9.011658 \\
\hline $\mathrm{C}$ & 1.903670 & 12.353617 & 10.169419 \\
\hline $\mathrm{C}$ & 2.339264 & 13.526262 & 7.262632 \\
\hline $\mathrm{C}$ & -0.212307 & 15.210590 & 6.486848 \\
\hline $\mathrm{C}$ & 4.615803 & 19.813154 & 6.660843 \\
\hline $\mathrm{C}$ & 5.876901 & 19.937597 & 7.531779 \\
\hline $\mathrm{C}$ & 4.710780 & 20.734996 & 5.421279 \\
\hline Th & 4.231719 & 21.100118 & 2.757567 \\
\hline $\mathrm{C}$ & 6.579068 & 19.584908 & 2.306903 \\
\hline $\mathrm{C}$ & 5.640988 & 7929 & 2.77 \\
\hline $\mathrm{C}$ & 4.645782 & 18.447266 & 1.768187 \\
\hline $\mathrm{C}$ & 4.973625 & 19.294745 & 0.673166 \\
\hline $\mathrm{C}$ & 6.169249 & 19.997748 & 1.002343 \\
\hline $\mathrm{C}$ & 3.252173 & 23.754906 & 2.272600 \\
\hline $\mathrm{C}$ & 3.647334 & 23.749984 & 3.640463 \\
\hline $\mathrm{C}$ & 5.071011 & 23.6738 & 596 \\
\hline $\mathrm{C}$ & 5.551897 & 23.616285 & 2.347911 \\
\hline $\mathrm{C}$ & 4.427194 & 23.655547 & 1.472832 \\
\hline $\mathrm{C}$ & 1.876492 & 24.061603 & 1.761098 \\
\hline $\mathrm{C}$ & 3.55 & 17.4 & 1.78 \\
\hline $\mathrm{C}$ & 4.319617 & 19.287992 & -0.676374 \\
\hline $\mathrm{C}$ & 2.730104 & 23.982312 & 4.807478 \\
\hline $\mathrm{C}$ & 5.798064 & 17.759425 & 3.993144 \\
\hline $\mathrm{C}$ & 6.996568 & 23.763113 & 1.966596 \\
\hline $\mathrm{C}$ & 6.996811 & 20.775900 & 0.019896 \\
\hline $\mathrm{C}$ & 5.944467 & 23.889267 & 4.889399 \\
\hline $\mathrm{C}$ & 7.879510 & 19.929536 & 2.976516 \\
\hline $\mathrm{C}$ & 4.458642 & 23.777337 & -0.023629 \\
\hline $\mathrm{H}$ & -1.709139 & 12.811257 & 11.071723 \\
\hline $\mathrm{H}$ & -1.335831 & 14.365097 & 11.808223 \\
\hline $\mathrm{H}$ & -0.215858 & 13.010943 & 11.984764 \\
\hline $\mathrm{H}$ & 3.520982 & 19.705467 & 12.383915 \\
\hline $\mathrm{H}$ & 1.830935 & 19.704868 & 11.882107 \\
\hline $\mathrm{H}$ & 3.101423 & 19.613862 & 10.669287 \\
\hline $\mathrm{H}$ & 3.246055 & 17.165597 & 2.804138 \\
\hline $\mathrm{H}$ & 3.891323 & 16.485914 & 1.309685 \\
\hline $\mathrm{H}$ & 2.665693 & 17.751585 & 1.243747 \\
\hline $\mathrm{H}$ & 5.880298 & 17.466167 & 11.390604 \\
\hline
\end{tabular}




\begin{tabular}{|c|c|c|c|}
\hline & 4.982399 & 18.284519 & 10.109887 \\
\hline & 5.462040 & 16.597938 & 9.912246 \\
\hline & -2.379127 & 15.986373 & 8.292469 \\
\hline & -2.497083 & 15.557026 & 9.998275 \\
\hline & -2.906500 & 14.371889 & 8.757971 \\
\hline & 3.781141 & 22.133443 & 7.717830 \\
\hline & 4.796380 & 14.279285 & 10.546846 \\
\hline & 3.992206 & 13.480754 & 11.901602 \\
\hline & 5.350623 & 14.564189 & 12.191384 \\
\hline & 0.003551 & 20.870989 & 9.266843 \\
\hline & 4.837877 & 17.471822 & 140 \\
\hline & 6.381248 & 18.255018 & 4.773889 \\
\hline & 6.327644 & 16.831061 & 3.739141 \\
\hline & 2.069596 & 12.644479 & 6.665620 \\
\hline & 3.261641 & 13.277363 & 7.797224 \\
\hline & 2.569487 & 14.338682 & 6.566760 \\
\hline & 2.025465 & 13.550509 & 13.013909 \\
\hline $\mathrm{H}$ & 0.700988 & 14.608259 & 13.508090 \\
\hline $\mathrm{H}$ & 2.221955 & 14.626323 & 14.392507 \\
\hline $\mathrm{H}$ & 4.566992 & 18.781723 & 6.298141 \\
\hline & 0.432911 & 17.422812 & 7.941675 \\
\hline $\mathrm{H}$ & 5.739739 & 20.632669 & 5.030371 \\
\hline $\mathrm{H}$ & 4.618817 & 21.779913 & 5.738001 \\
\hline $\mathrm{H}$ & -1.585057 & 19.683414 & 8.127775 \\
\hline $\mathrm{H}$ & -1.930959 & 18.009537 & 7.698112 \\
\hline $\mathrm{H}$ & -0.879233 & 18.992464 & 6.655153 \\
\hline $\mathrm{H}$ & 3.080538 & 23.491199 & 5.719513 \\
\hline $\mathrm{H}$ & 1.721655 & 23.606398 & 4.611006 \\
\hline $\mathrm{H}$ & 2.637125 & 25.054063 & 5.028566 \\
\hline $\mathrm{H}$ & -1.317696 & 17.617472 & 10.049762 \\
\hline $\mathrm{H}$ & -0.082744 & 18.734566 & 10.652391 \\
\hline $\mathrm{H}$ & 5.984314 & 20.953866 & 7.927782 \\
\hline $\mathrm{H}$ & 6.777353 & 19.713513 & 6.947886 \\
\hline $\mathrm{H}$ & 5.849828 & 19.248106 & 8.381656 \\
\hline $\mathrm{H}$ & 2.484038 & 23.695401 & 8.753075 \\
\hline $\mathrm{H}$ & -0.531737 & 23.146224 & 8.775992 \\
\hline $\mathrm{H}$ & 0.406568 & 23.655657 & 7.367060 \\
\hline $\mathrm{F}$ & 0.242525 & 24.734463 & 8.765729 \\
\hline X & 7.341438 & 24.786647 & 2.167024 \\
\hline $\mathrm{H}$ & 7.162985 & 23.572814 & 0.904368 \\
\hline $\mathrm{H}$ & 7.653919 & 23.094683 & 2.533236 \\
\hline $\mathrm{H}$ & 1.616195 & 11.360321 & 9.800203 \\
\hline $\mathrm{H}$ & 1.795347 & 12.332643 & 11.256585 \\
\hline $\mathrm{H}$ & 2.966680 & 12.479229 & 9.945137 \\
\hline
\end{tabular}




\begin{tabular}{lrrc}
$\mathrm{H}$ & 0.673743 & 15.653916 & 6.021318 \\
$\mathrm{H}$ & -0.995422 & 15.973036 & 6.501608 \\
$\mathrm{H}$ & -0.561807 & 14.403031 & 5.830235 \\
$\mathrm{H}$ & 1.735447 & 25.147867 & 1.676147 \\
$\mathrm{H}$ & 1.092861 & 23.686654 & 2.426030 \\
$\mathrm{H}$ & 1.697908 & 23.629376 & 0.773583 \\
$\mathrm{H}$ & 3.672529 & 23.183974 & -0.501696 \\
$\mathrm{H}$ & 5.416398 & 23.456069 & -0.441729 \\
$\mathrm{H}$ & 4.307825 & 24.819137 & -0.336524 \\
$\mathrm{H}$ & 0.734799 & 22.580870 & 11.008227 \\
$\mathrm{H}$ & 1.445935 & 24.199568 & 10.949313 \\
$\mathrm{H}$ & 2.492731 & 22.772643 & 11.066263 \\
$\mathrm{H}$ & -0.123723 & 16.818489 & 13.352665 \\
$\mathrm{H}$ & 0.367333 & 18.470899 & 12.995480 \\
$\mathrm{H}$ & 1.029205 & 17.639290 & 14.403996 \\
$\mathrm{H}$ & 6.764455 & 23.166231 & 4.962487 \\
$\mathrm{H}$ & 5.376805 & 23.833347 & 5.821594 \\
$\mathrm{H}$ & 6.400979 & 24.887638 & 4.853639 \\
$\mathrm{H}$ & 4.385365 & 20.261753 & -1.171054 \\
$\mathrm{H}$ & 3.261415 & 19.023611 & -0.617848 \\
$\mathrm{H}$ & 4.805998 & 18.557188 & -1.336971 \\
$\mathrm{H}$ & 7.561271 & 20.088826 & -0.625132 \\
$\mathrm{H}$ & 7.726934 & 21.422994 & 0.510460 \\
$\mathrm{H}$ & 6.387442 & 21.397944 & -0.642732 \\
$\mathrm{H}$ & 7.790372 & 19.966582 & 4.067452 \\
$\mathrm{H}$ & 8.271001 & 20.896632 & 2.647328 \\
$\mathrm{H}$ & 8.649516 & 19.180485 & 2.747668 \\
$\mathrm{C}$ & 2.240428 & 19.944634 & 4.188886 \\
$\mathrm{H}$ & 3.504909 & 20.318610 & 4.729243 \\
$\mathrm{H}$ & 2.036057 & 19.227973 & 3.386314 \\
$\mathrm{H}$ & 1.505339 & 20.755823 & 4.102706 \\
$\mathrm{H}$ & 2.036538 & 19.427061 & 5.131487 \\
$\mathrm{H}$ & 2.231397 & 20.910271 & 1.269057 \\
$\mathrm{H}$ & 2.379380 & 21.350347 & 0.270965 \\
& 1.355517 & 19.865102 & 1.103701 \\
\hline
\end{tabular}

\section{Int3}

$\mathrm{E}=-2263.028745$

$\mathrm{H}=-2261.694293$

$\begin{array}{llll}\text { Th } & -0.922705 & -1.485814 & 2.622811\end{array}$ 


\begin{tabular}{|c|c|c|c|}
\hline $\mathrm{C}$ & -3.147873 & -2.449435 & 1.100389 \\
\hline $\mathrm{C}$ & -2.108552 & -3.410399 & 0.941613 \\
\hline $\mathrm{C}$ & -1.919086 & -4.068752 & 2.192445 \\
\hline $\mathrm{C}$ & -2.837343 & -3.504955 & 3.129278 \\
\hline $\mathrm{C}$ & -3.593885 & -2.506332 & 2.453926 \\
\hline $\mathrm{C}$ & 0.517568 & -2.649306 & 4.784898 \\
\hline $\mathrm{C}$ & 1.436970 & -2.584460 & 3.697400 \\
\hline $\mathrm{C}$ & 1.750852 & -1.211909 & 3.471156 \\
\hline $\mathrm{C}$ & 1.014100 & -0.428919 & 4.405634 \\
\hline $\mathrm{C}$ & 0.234944 & -1.313770 & 5.203472 \\
\hline $\mathrm{P}$ & 0.011844 & -0.102070 & 0.065596 \\
\hline $\mathrm{C}$ & -0.013311 & 1.486717 & 1.084220 \\
\hline $\mathrm{C}$ & -1.088377 & 1.889249 & 1.917446 \\
\hline $\mathrm{C}$ & -1.018540 & 3.111515 & 2.596306 \\
\hline $\mathrm{C}$ & 0.074722 & 3.964746 & 2.478947 \\
\hline $\mathrm{C}$ & 1.138105 & 3.541985 & 1.678300 \\
\hline $\mathrm{C}$ & 1.131040 & 2.323922 & 0.993373 \\
\hline $\mathrm{C}$ & 0.121915 & 5.305243 & 3.191100 \\
\hline $\mathrm{C}$ & -0.971121 & 6.254338 & 2.679731 \\
\hline $\mathrm{C}$ & 0.048505 & 5.153854 & 4.716331 \\
\hline $\mathrm{C}$ & -2.340594 & 1.044366 & 2.154305 \\
\hline $\mathrm{C}$ & -3.637348 & 1.826741 & 1.887128 \\
\hline $\mathrm{C}$ & -2.295621 & 0.351316 & 3.533649 \\
\hline $\mathrm{C}$ & 2.317686 & 1.949046 & 0.116154 \\
\hline $\mathrm{C}$ & 1.992283 & 2.234503 & -1.364617 \\
\hline Th & 0.769162 & 0.792575 & -3.086290 \\
\hline $\mathrm{C}$ & -1.091511 & 1.850900 & -4.977477 \\
\hline $\mathrm{C}$ & -1.710353 & 2.079360 & -3.716801 \\
\hline $\mathrm{C}$ & -0.957709 & 3.075426 & -3.026933 \\
\hline $\mathrm{C}$ & 0.118534 & 3.472651 & -3.867096 \\
\hline $\mathrm{C}$ & 0.040038 & 2.715145 & -5.071723 \\
\hline $\mathrm{C}$ & 3.350250 & -0.445418 & -2.998824 \\
\hline $\mathrm{C}$ & 2.456743 & -1.547635 & -3.128105 \\
\hline $\mathrm{C}$ & 1.886170 & -1.506459 & -4.428994 \\
\hline $\mathrm{C}$ & 2.411480 & -0.367217 & -5.102491 \\
\hline $\mathrm{C}$ & 3.315548 & 0.294173 & -4.214544 \\
\hline $\mathrm{C}$ & 1.179273 & 1.043573 & 4.634588 \\
\hline $\mathrm{C}$ & -4.829248 & -1.859796 & 3.005014 \\
\hline $\mathrm{C}$ & -1.450008 & -3.828917 & -0.340211 \\
\hline $\mathrm{C}$ & -0.584814 & -0.935216 & 6.402968 \\
\hline $\mathrm{C}$ & 4.325247 & -0.249055 & -1.877704 \\
\hline $\mathrm{C}$ & 2.864109 & -0.723749 & 2.593776 \\
\hline $\mathrm{C}$ & 0.176962 & -3.876474 & 5.578566 \\
\hline $\mathrm{C}$ & -1.351523 & 3.698729 & -1.719566 \\
\hline
\end{tabular}




\begin{tabular}{|c|c|c|c|}
\hline $\mathrm{C}$ & 1.059569 & 4.622473 & -3.651905 \\
\hline $\mathrm{C}$ & 2.298794 & -2.660902 & -2.133781 \\
\hline $\mathrm{C}$ & -3.833129 & -1.697854 & -0.004375 \\
\hline $\mathrm{C}$ & -3.040511 & 1.544182 & -3.276018 \\
\hline $\mathrm{C}$ & 2.272429 & -0.112049 & -6.575846 \\
\hline $\mathrm{C}$ & -1.105741 & -5.320078 & 2.355697 \\
\hline $\mathrm{C}$ & 0.821822 & 3.032311 & -6.313693 \\
\hline $\mathrm{C}$ & -3.162229 & -4.018008 & 4.502739 \\
\hline $\mathrm{C}$ & 1.043770 & -2.571919 & -5.065261 \\
\hline $\mathrm{C}$ & 2.156407 & -3.737288 & 3.056130 \\
\hline $\mathrm{C}$ & -1.647856 & 0.994744 & -6.078360 \\
\hline $\mathrm{C}$ & 3.625749 & 2.620823 & 0.563834 \\
\hline $\mathrm{C}$ & 4.222695 & 1.437977 & -4.570133 \\
\hline $\mathrm{H}$ & -5.695322 & -2.523753 & 2.877813 \\
\hline $\mathrm{H}$ & -5.069221 & -0.922635 & 2.497055 \\
\hline $\mathrm{H}$ & -4.743435 & -1.644072 & 4.073955 \\
\hline $\mathrm{H}$ & 3.831700 & -0.863726 & 3.095153 \\
\hline $\mathrm{H}$ & 2.764050 & 0.339435 & 2.372213 \\
\hline $\mathrm{H}$ & 2.914256 & -1.260648 & 1.642060 \\
\hline $\mathrm{H}$ & -1.740052 & 2.961262 & -1.010429 \\
\hline $\mathrm{H}$ & -2.136508 & 4.453300 & -1.865822 \\
\hline $\mathrm{H}$ & -0.509784 & 4.192092 & -1.227805 \\
\hline $\mathrm{H}$ & 3.181526 & -3.814316 & 3.441834 \\
\hline $\mathrm{H}$ & 2.239919 & -3.629106 & 1.968635 \\
\hline $\mathrm{H}$ & 1.664354 & -4.691297 & 3.258102 \\
\hline $\mathrm{H}$ & -3.177425 & -1.537162 & -0.863004 \\
\hline $\mathrm{H}$ & -4.202692 & -0.721112 & 0.321115 \\
\hline $\mathrm{H}$ & -4.702617 & -2.264479 & -0.362764 \\
\hline $\mathrm{H}$ & 2.003477 & 4.192120 & 1.587674 \\
\hline $\mathrm{H}$ & 0.173091 & -4.785215 & 4.972769 \\
\hline $\mathrm{H}$ & -0.791895 & -3.797431 & 6.076512 \\
\hline $\mathrm{H}$ & 0.927435 & -4.024240 & 6.366692 \\
\hline $\mathrm{H}$ & -1.848756 & 3.395671 & 3.238709 \\
\hline $\mathrm{H}$ & -3.084073 & 1.388905 & -2.193115 \\
\hline $\mathrm{H}$ & -3.276976 & 0.589731 & -3.752922 \\
\hline $\mathrm{H}$ & -3.844720 & 2.247800 & -3.532187 \\
\hline $\mathrm{H}$ & -1.630584 & -6.170463 & 1.900294 \\
\hline $\mathrm{H}$ & -0.937321 & -5.574837 & 3.403829 \\
\hline $\mathrm{H}$ & -0.130433 & -5.254503 & 1.863997 \\
\hline $\mathrm{H}$ & -1.427449 & -1.616629 & 6.557545 \\
\hline $\mathrm{H}$ & -0.994999 & 0.073666 & 6.312725 \\
\hline $\mathrm{H}$ & 0.021658 & -0.961672 & 7.318265 \\
\hline $\mathrm{H}$ & 2.469963 & 0.867144 & 0.234153 \\
\hline $\mathrm{H}$ & -2.334097 & 0.267779 & 1.353607 \\
\hline
\end{tabular}




$\begin{array}{rrrr}\mathrm{H} & 2.955149 & 2.317744 & -1.906018 \\ \mathrm{H} & 1.577891 & 3.251288 & -1.410052 \\ \mathrm{H} & -3.772290 & 2.624071 & 2.623679 \\ \mathrm{H} & -4.506781 & 1.166879 & 1.966128 \\ \mathrm{H} & -3.639164 & 2.280557 & 0.890161 \\ \mathrm{H} & 1.346003 & -3.182552 & -2.259598 \\ \mathrm{H} & 2.340358 & -2.299344 & -1.101776 \\ \mathrm{H} & 3.095606 & -3.408436 & -2.251282 \\ \mathrm{H} & -3.306016 & 0.116302 & 3.884138 \\ \mathrm{H} & -1.832311 & 1.010282 & 4.274307 \\ \mathrm{H} & 3.627298 & 3.692419 & 0.334539 \\ \mathrm{H} & 4.466432 & 2.181768 & 0.018444 \\ \mathrm{H} & 3.824886 & 2.501826 & 1.636786 \\ \mathrm{H} & 1.092519 & 5.760374 & 2.951549 \\ \mathrm{H} & -1.970245 & 5.861431 & 2.898514 \\ \mathrm{H} & -0.899214 & 6.393842 & 1.596782 \\ \mathrm{H} & -0.888000 & 7.236997 & 3.157397 \\ \mathrm{H} & 2.878393 & -0.830547 & -7.144346 \\ \mathrm{H} & 2.615864 & 0.886051 & -6.854880 \\ \mathrm{H} & 1.241999 & -0.220956 & -6.929394 \\ \mathrm{H} & -4.145896 & -4.505074 & 4.502293 \\ \mathrm{H} & -3.206950 & -3.221605 & 5.254443 \\ \mathrm{H} & -2.437376 & -4.758578 & 4.844460 \\ \mathrm{H} & -0.360855 & -3.908134 & -0.248833 \\ \mathrm{H} & -1.655146 & -3.128856 & -1.151677 \\ \mathrm{H} & -1.815779 & -4.815912 & -0.653925 \\ \mathrm{H} & 5.212575 & -0.880966 & -2.021998 \\ \mathrm{H} & 3.900505 & -0.510986 & -0.903849 \\ \mathrm{H} & 4.674024 & 0.784794 & -1.816807 \\ \mathrm{H} & 4.432979 & 2.081461 & -3.710056 \\ \mathrm{H} & 3.798432 & 2.071577 & -5.354882 \\ \mathrm{H} & 5.190442 & 1.075275 & -4.942480 \\ \mathrm{H} & -0.905109 & 4.712310 & 5.026474 \\ \mathrm{H} & 0.135701 & 6.129333 & 5.207726 \\ \mathrm{H} & 0.851728 & 4.511702 & 5.090551 \\ \mathrm{H} & 0.304568 & 1.481826 & 5.120636 \\ \mathrm{H} & 1.347953 & 1.595904 & 3.707136 \\ \mathrm{H} & 2.040604 & 1.227875 & 5.290732 \\ \mathrm{H} & 0.378378 & -2.164363 & -5.832752 \\ \mathrm{H} & 0.416393 & -3.092407 & -4.337118 \\ \mathrm{H} & 1.674992 & -3.326272 & -5.555122 \\ \mathrm{H} & 2.099003 & 4.367989 & -3.888113 \\ \mathrm{H} & 1.040288 & 4.9777712 & -2.619086 \\ & & 5.471749 & -4.291890\end{array}$




$\begin{array}{llll}\mathrm{H} & 0.432301 & 3.946133 & -6.783467 \\ \mathrm{H} & 0.757955 & 2.238073 & -7.060141 \\ \mathrm{H} & 1.881732 & 3.214889 & -6.107532 \\ \mathrm{H} & -2.129319 & 0.092612 & -5.689851 \\ \mathrm{H} & -0.875246 & 0.676389 & -6.784769 \\ \mathrm{H} & -2.403986 & 1.540165 & -6.659341 \\ \mathrm{H} & -1.368187 & -0.075969 & -0.318936 \\ \mathrm{C} & -0.975493 & -1.018265 & -2.967470 \\ \mathrm{H} & -0.579488 & -1.918555 & -2.479488 \\ \mathrm{H} & -1.276152 & -1.322624 & -3.982211 \\ \mathrm{H} & -1.895816 & -0.745112 & -2.438564\end{array}$
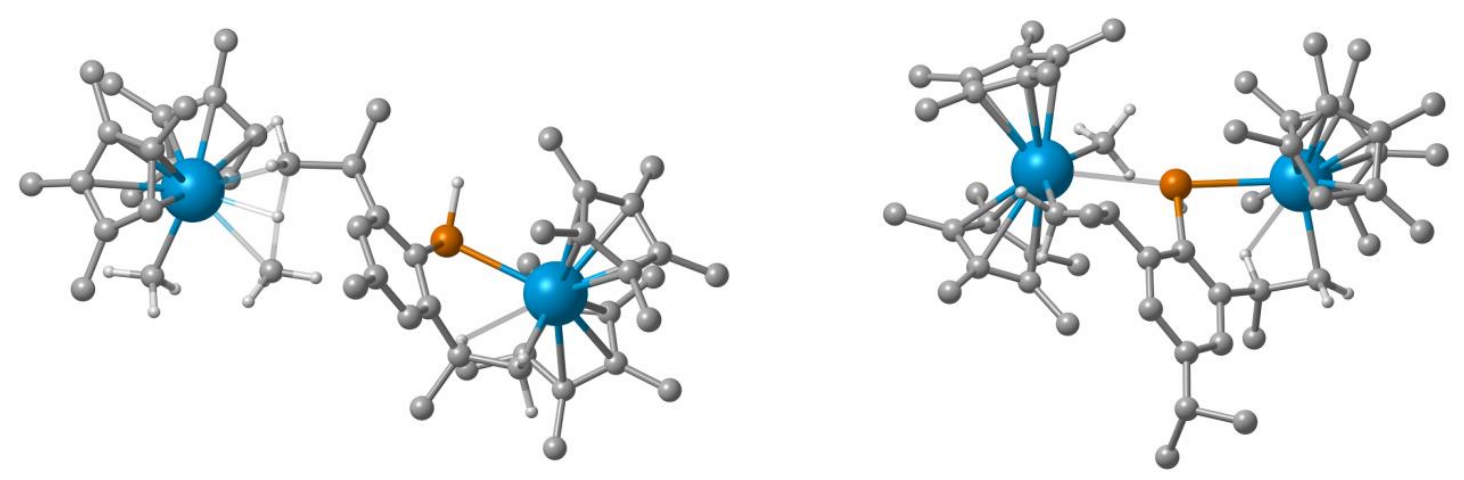

Figure S9. Optimized structures of TS3 (left) and Int3 (right). Some methyl carbons and the majority of hydrogen atoms have been omitted for clarity.

\section{TS4}

\begin{tabular}{lrrr}
$\mathrm{E}=-2263.001780$ & \\
$\mathrm{H}=-2261.669570$ & & \\
\multicolumn{4}{l}{} \\
Th & 1.774571 & 16.330724 & 9.223951 \\
$\mathrm{C}$ & -0.489655 & 15.228517 & 7.813786 \\
$\mathrm{C}$ & 0.567749 & 14.286765 & 7.673604 \\
$\mathrm{C}$ & 0.805836 & 13.693999 & 8.949071 \\
$\mathrm{C}$ & -0.109106 & 14.273786 & 9.878242 \\
$\mathrm{C}$ & -0.897907 & 15.227351 & 9.179070 \\
$\mathrm{C}$ & 3.185257 & 15.248920 & 11.474981 \\
$\mathrm{C}$ & 4.143258 & 15.365934 & 10.425081 \\
$\mathrm{C}$ & 4.399656 & 16.753319 & 10.218818 \\
$\mathrm{C}$ & 3.571704 & 17.492918 & 11.107841 \\
$\mathrm{C}$ & 2.806107 & 16.565036 & 11.872110
\end{tabular}



$\begin{array}{llll}\text { P } & 2.937047 & 17.692933 & 6.848343\end{array}$
$\begin{array}{llll}\text { C } & 2.737509 & 19.362515 & 7.714715\end{array}$
$\begin{array}{llll}\text { C } & 1.578161 & 19.740788 & 8.443668\end{array}$
$\begin{array}{llll}\text { C } & 1.529841 & 20.981903 & 9.090413\end{array}$
$\begin{array}{llll}\text { C } & 2.586345 & 21.886601 & 9.044291\end{array}$
$\begin{array}{llll}\text { C } & 3.728837 & 21.502863 & 8.337479\end{array}$
$\begin{array}{llll}\text { C } & 3.835871 & 20.272338 & 7.685103\end{array}$
$\begin{array}{llll}\text { C } & 2.513907 & 23.236124 & 9.736768\end{array}$
$\begin{array}{llll}\text { C } & 1.397387 & 24.115579 & 9.157116\end{array}$
$\begin{array}{llll}\text { C } & 2.366876 & 23.095702 & 11.257997\end{array}$
$\begin{array}{llll}\text { C } & 0.361541 & 18.834202 & 8.613032\end{array}$
$\begin{array}{llll}\text { C } & -0.960405 & 19.534932 & 8.262881\end{array}$
$\begin{array}{llll}\text { C } & 0.352314 & 18.175084 & 10.011231\end{array}$
$\begin{array}{llll}\text { C } & 5.095976 & 19.946108 & 6.903754\end{array}$
$\begin{array}{llll}\text { C } & 4.838702 & 20.182150 & 5.403811\end{array}$
$\begin{array}{llll}\text { Th } & 3.361705 & 18.746858 & 3.940458\end{array}$
$\begin{array}{llll}\text { C } & 1.519533 & 19.891232 & 2.065868\end{array}$
$\begin{array}{llll}\text { C } & 0.999572 & 20.239230 & 3.347745\end{array}$
$\begin{array}{llll}\text { C } & 1.877824 & 21.193665 & 3.938486\end{array}$
$\begin{array}{llll}\text { C } & 2.933758 & 21.444670 & 3.018501\end{array}$
$\begin{array}{llll}\text { C } & 2.711550 & 20.645503 & 1.861795\end{array}$
$\begin{array}{llll}\text { C } & 5.793068 & 17.247511 & 3.935557\end{array}$
$\begin{array}{llll}\text { C } & 4.782878 & 16.250001 & 3.802832\end{array}$
$\begin{array}{llll}\text { C } & 4.190368 & 16.387652 & 2.517756\end{array}$
$\begin{array}{llll}\text { C } & 4.826696 & 17.475515 & 1.854699\end{array}$
$\begin{array}{llll}\text { C } & 5.818058 & 18.010558 & 2.735347\end{array}$
$\begin{array}{llll}\text { C } & 3.643118 & 18.972847 & 11.341761\end{array}$
$\begin{array}{llll}\text { C } & -2.134445 & 15.874924 & 9.725800\end{array}$
$\begin{array}{llll}\text { C } & 1.198184 & 13.802661 & 6.401211\end{array}$
$\begin{array}{llll}\text { C } & 1.914842 & 16.906745 & 13.030860\end{array}$
$\begin{array}{llll}\text { C } & 6.755311 & 17.334184 & 5.080427\end{array}$
$\begin{array}{llll}\text { C } & 5.536536 & 17.299063 & 9.411010\end{array}$
$\begin{array}{llll}\text { C } & 2.908717 & 14.004923 & 12.267046\end{array}$
$\begin{array}{llll}\text { C } & 1.620565 & 21.929860 & 5.219637\end{array}$
$\begin{array}{llll}\text { C } & 3.972428 & 22.525055 & 3.109082\end{array}$
$\begin{array}{llll}\text { C } & 4.554241 & 15.123970 & 4.766707\end{array}$
$\begin{array}{llll}\text { C } & -1.240214 & 15.902129 & 6.702703\end{array}$
$\begin{array}{llll}\text { C } & -0.354942 & 19.878472 & 3.884715\end{array}$
$\begin{array}{llll}\text { C } & 4.728560 & 17.742328 & 0.380718\end{array}$
$\begin{array}{llll}\text { C } & 1.656561 & 12.471670 & 9.144286\end{array}$
$\begin{array}{llll}\text { C } & 3.419648 & 20.848884 & 0.553862\end{array}$
$\begin{array}{llll}\text { C } & -0.405708 & 13.812685 & 11.275900\end{array}$
$\begin{array}{llll}\text { C } & 3.230453 & 15.434068 & 1.867831\end{array}$
$\begin{array}{llll}\text { C } & 4.946576 & 14.252864 & 9.814128\end{array}$ 


\begin{tabular}{|c|c|c|c|}
\hline $\mathrm{C}$ & 0.819845 & 19.066723 & 1.022232 \\
\hline $\mathrm{C}$ & 6.343945 & 20.691860 & 7.397964 \\
\hline C & 6.825060 & 19.064484 & 2.371512 \\
\hline $\mathrm{H}$ & -2.992706 & 15.193689 & 9.641064 \\
\hline H & -2.397386 & 16.788153 & 9.185764 \\
\hline & -2.032791 & 16.134309 & 10.783683 \\
\hline F & 6.483084 & 17.164534 & 9.953134 \\
\hline $\mathrm{H}$ & 5.415495 & 18.366135 & 9.221043 \\
\hline $\mathrm{H}$ & 5.634521 & 16.801702 & 8.442653 \\
\hline F & 1.160470 & 21.294001 & 5.981145 \\
\hline $\mathrm{H}$ & 0.943725 & 22.778584 & 5.050425 \\
\hline $\mathrm{H}$ & 2.540545 & 22.323372 & 5.657508 \\
\hline $\mathrm{H}$ & 5.953693 & 14.214078 & 10.250515 \\
\hline $\mathrm{H}$ & 5.079414 & 14.378453 & 8.733482 \\
\hline $\mathrm{H}$ & 4.486653 & 13.275808 & 9.981764 \\
\hline $\mathrm{H}$ & -0.686426 & 15.869816 & 5.764127 \\
\hline $\mathrm{H}$ & -1.467435 & 16.950692 & 6.915850 \\
\hline $\mathrm{H}$ & -2.199497 & 15.394920 & 6.534509 \\
\hline $\mathrm{H}$ & 4.567696 & 22.193107 & 8.302563 \\
\hline $\mathrm{H}$ & 2.938022 & 13.097053 & 11.660444 \\
\hline $\mathrm{H}$ & 1.944530 & 14.040329 & 12.777773 \\
\hline $\mathrm{H}$ & 3.675602 & 13.889435 & 13.045057 \\
\hline I & 0.636430 & 21.235971 & 9.656292 \\
\hline $\mathrm{H}$ & -0.343509 & 19.707620 & 4.965388 \\
\hline $\mathrm{H}$ & -0.760395 & 18.979661 & 3.413773 \\
\hline I & -1.068689 & 20.691627 & 3.696066 \\
\hline $\mathrm{H}$ & 1.196567 & 11.608655 & 8.644497 \\
\hline $\mathrm{H}$ & 1.764775 & 12.208042 & 10.197861 \\
\hline $\mathrm{H}$ & 2.661117 & 12.577900 & 8.721575 \\
\hline $\mathrm{H}$ & 1.134717 & 16.155195 & 13.186261 \\
\hline $\mathrm{H}$ & 1.415447 & 17.868704 & 12.888998 \\
\hline $\mathrm{H}$ & 2.488326 & 16.971358 & 13.965474 \\
\hline $\mathrm{H}$ & 5.277198 & 18.874218 & 7.046293 \\
\hline $\mathrm{H}$ & 0.486601 & 18.053413 & 7.818220 \\
\hline $\mathrm{H}$ & 5.809962 & 20.120945 & 4.878185 \\
\hline $\mathrm{H}$ & 4.534815 & 21.231747 & 5.291065 \\
\hline $\mathrm{H}$ & -1.189354 & 20.323835 & 8.985633 \\
\hline $\mathrm{H}$ & -1.791662 & 18.823352 & 8.293179 \\
\hline 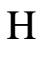 & -0.933535 & 19.986334 & 7.265023 \\
\hline $\mathrm{H}$ & 3.590723 & 14.636551 & 4.596945 \\
\hline $\mathrm{H}$ & 4.573488 & 15.469263 & 5.805270 \\
\hline $\mathrm{H}$ & 5.329719 & 14.354035 & 4.653191 \\
\hline $\mathrm{H}$ & -0.668641 & 17.925944 & 10.317494 \\
\hline 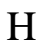 & 0.761208 & 18.864179 & 10.755784 \\
\hline
\end{tabular}




$\begin{array}{cccc}\text { H } & 6.306989 & 21.760592 & 7.155726 \\ \mathrm{H} & 7.230182 & 20.287426 & 6.897222 \\ \mathrm{H} & 6.498888 & 20.593996 & 8.479978 \\ \mathrm{H} & 3.468179 & 23.745428 & 9.544167 \\ \mathrm{H} & 0.411866 & 23.669227 & 9.329958 \\ \mathrm{H} & 1.517176 & 24.247864 & 8.077506 \\ \mathrm{H} & 1.399567 & 25.106511 & 9.625168 \\ \mathrm{H} & 5.301776 & 16.986917 & -0.174213 \\ \mathrm{H} & 5.136534 & 18.716942 & 0.107502 \\ \mathrm{H} & 3.700974 & 17.692505 & 0.007497 \\ \mathrm{H} & -1.406668 & 13.364510 & 11.320315 \\ \mathrm{H} & -0.395536 & 14.629210 & 12.007093 \\ \mathrm{H} & 0.301039 & 13.053695 & 11.614430 \\ \mathrm{H} & 2.290906 & 13.759728 & 6.462569 \\ \mathrm{H} & 0.943800 & 14.443227 & 5.555532 \\ \mathrm{H} & 0.852237 & 12.788001 & 6.161757 \\ \mathrm{H} & 7.557637 & 16.591843 & 4.971492 \\ \mathrm{H} & 6.255004 & 17.142668 & 6.034477 \\ \mathrm{H} & 7.229121 & 18.316764 & 5.148598 \\ \mathrm{H} & 7.270014 & 19.526484 & 3.256955 \\ \mathrm{H} & 6.389144 & 19.868995 & 1.769084 \\ \mathrm{H} & 7.647064 & 18.636080 & 1.782543 \\ \mathrm{H} & 1.421747 & 22.608299 & 11.522081 \\ \mathrm{H} & 2.378533 & 24.078718 & 11.742355 \\ \mathrm{H} & 3.180003 & 22.497983 & 11.681101 \\ \mathrm{H} & 2.737216 & 19.352358 & 11.820336 \\ \mathrm{H} & 3.785872 & 19.537939 & 10.417375 \\ \mathrm{H} & 4.483600 & 19.209834 & 12.008112 \\ \mathrm{H} & 2.561367 & 15.937525 & 1.162329 \\ \mathrm{H} & 2.603006 & 14.913867 & 2.596346 \\ \mathrm{H} & 3.771958 & 14.665238 & 1.300118 \\ \mathrm{H} & 4.982513 & 22.166705 & 2.881503 \\ \mathrm{H} & 4.005797 & 22.975201 & 4.103580 \\ \mathrm{H} & 3.748412 & 23.330099 & 2.396293 \\ \mathrm{H} & 3.093021 & 21.793163 & 0.096516 \\ \mathrm{H} & 3.202431 & 20.055328 & -0.163537 \\ \mathrm{H} & 4.507454 & 20.915594 & 0.661810 \\ \mathrm{H} & 0.112545 & 18.358802 & 1.464055 \\ \mathrm{H} & 1.518188 & 18.489304 & 0.407392 \\ \mathrm{H} & 0.245962 & 19.705039 & 0.336993 \\ \mathrm{H} & 1.925120 & 17.595809 & 5.471259 \\ \mathrm{C} & 1.299314 & 17.082237 & 4.198065 \\ \mathrm{H} & 1.554977 & 16.032262 & 4.367558 \\ \mathrm{H} & 1.238233 & 17.222149 & 3.103809\end{array}$


H $\quad 0.290440 \quad 17.259661 \quad 4.576086$

\section{Prod}

$\mathrm{E}=-2222.556736$

$\mathrm{H}=-2221.268584$

$\begin{array}{llll}\text { Th } & -0.828110 & -1.464443 & 2.333359\end{array}$

$\begin{array}{llll}\text { C } & -3.050140 & -2.351144 & 0.743726\end{array}$

$\begin{array}{llll}\text { C } & -2.002152 & -3.286670 & 0.516371\end{array}$

$\begin{array}{llll}\text { C } & -1.809682 & -4.038145 & 1.711530\end{array}$

$\begin{array}{llll}\text { C } & -2.738608 & -3.555398 & 2.682879\end{array}$

C $\quad-3.505908 \quad-2.516939 \quad 2.081263$

$\begin{array}{llll}\text { C } & 0.482939 & -2.699659 & 4.568768\end{array}$

$\begin{array}{llll}\text { C } & 1.467392 & -2.569487 & 3.543716\end{array}$

$\begin{array}{llll}\text { C } & 1.768835 & -1.183457 & 3.400163\end{array}$

$\begin{array}{llll}\text { C } & 0.949069 & -0.456937 & 4.308149\end{array}$

$\begin{array}{llll}\text { C } & 0.143428 & -1.391820 & 5.020763\end{array}$

$\begin{array}{llll}\mathrm{P} & 0.185796 & -0.079597 & 0.013494\end{array}$

$\begin{array}{llll}\text { C } & 0.129565 & 1.598784 & 0.913767\end{array}$

$\begin{array}{llll}\text { C } & -1.004140 & 2.002255 & 1.683277\end{array}$

$\begin{array}{llll}\text { C } & -0.996330 & 3.226384 & 2.359567\end{array}$

C $\quad 0.078509 \quad 4.109380 \quad 2.297786$

C $\quad 1.185986 \quad 3.709475 \quad 1.550421$

C $\quad 1.253404 \quad 2.484556 \quad 0.879246$

$\begin{array}{llll}\text { C } & 0.061719 & 5.444461 & 3.020117\end{array}$

C $\quad-1.053046 \quad 6.362505 \quad 2.499963$

$\begin{array}{llll}\text { C } & -0.035669 & 5.271334 & 4.542066\end{array}$

C $\quad-2.253525 \quad 1.138266 \quad 1.856280$

$\begin{array}{llll}\text { C } & -3.560916 & 1.924946 & 1.660284\end{array}$

$\begin{array}{llll}\text { C } & -2.226246 & 0.370189 & 3.202155\end{array}$

$\begin{array}{llll}\text { C } & 2.543133 & 2.159897 & 0.123958\end{array}$

C $\quad 2.444153 \quad 2.557698 \quad-1.372544$

$\begin{array}{llll}\text { Th } & 0.943723 & 0.956735 & -2.539910\end{array}$

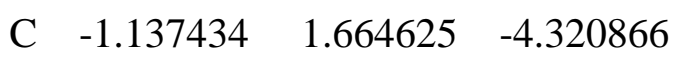

$\begin{array}{llll}\text { C } & -1.559385 & 2.123081 & -3.035371\end{array}$

$\begin{array}{llll}\text { C } & -0.751514 & 3.238114 & -2.676714\end{array}$

$\begin{array}{llll}\text { C } & 0.174767 & 3.467084 & -3.733842\end{array}$

$\begin{array}{llll}\text { C } & -0.078624 & 2.513031 & -4.758764\end{array}$

C $3.458588 \quad-0.412842 \quad-2.691867$

C $\quad 2.500473 \quad-1.455793 \quad-2.525002$

C $\quad 1.709451 \quad-1.515609 \quad-3.706169$

$\begin{array}{llll}\text { C } & 2.190179 & -0.526039 & -4.612970\end{array}$

C $\quad 3.265625 \quad 0.164753 \quad-3.978114$ 


\begin{tabular}{|c|c|c|c|}
\hline $\mathrm{C}$ & 1.062633 & 1.009585 & 828 \\
\hline $\mathrm{C}$ & -4.739852 & -1.912475 & 2.682270 \\
\hline $\mathrm{C}$ & -1.360081 & -3.557846 & -0.810944 \\
\hline $\mathrm{C}$ & -0.767418 & -1.070717 & 6.169886 \\
\hline $\mathrm{C}$ & 4.603971 & -0.133572 & -1.765490 \\
\hline $\mathrm{C}$ & 2.911246 & -0.622771 & 2.610314 \\
\hline $\mathrm{C}$ & 0.127263 & -3.971342 & 5.281814 \\
\hline $\mathrm{C}$ & -0.963492 & 4.123099 & -1.485094 \\
\hline $\mathrm{C}$ & 1.108424 & 4.634241 & -3.866565 \\
\hline $\mathrm{C}$ & 2.499037 & -2.452399 & -1.405530 \\
\hline $\mathrm{C}$ & -3.684093 & -1.505357 & -0.319549 \\
\hline $\mathrm{C}$ & -2.771938 & 1.644666 & -2.294021 \\
\hline $\mathrm{C}$ & 1.864101 & -0.498389 & -6.077493 \\
\hline $\mathrm{C}$ & -0.957511 & -5.270388 & 1.816141 \\
\hline $\mathrm{C}$ & 0.492255 & 2.603786 & -6.143901 \\
\hline $\mathrm{C}$ & -3.071379 & -4.180558 & 4.007145 \\
\hline $\mathrm{C}$ & 0.679941 & -2.555386 & -4.046143 \\
\hline $\mathrm{C}$ & 2.242719 & -3.686756 & 2.904806 \\
\hline $\mathrm{C}$ & -1.846306 & 0.617971 & -5.134137 \\
\hline $\mathrm{C}$ & 3.798014 & 2.769854 & 0.771464 \\
\hline $\mathrm{C}$ & 4.153760 & 1.193151 & -4.617126 \\
\hline $\mathrm{H}$ & -5.605820 & -2.569825 & 2.523411 \\
\hline $\mathrm{H}$ & -4.985933 & -0.945405 & 2.236549 \\
\hline $\mathrm{H}$ & -4.644826 & -1.761250 & 3.761744 \\
\hline $\mathrm{H}$ & 3.848300 & -0.704001 & 3.178995 \\
\hline $\mathrm{H}$ & 2.754178 & 0.431345 & 2.378294 \\
\hline $\mathrm{H}$ & 3.050219 & -1.147395 & 1.661827 \\
\hline $\mathrm{H}$ & -1.330644 & 3.570350 & -0.616920 \\
\hline $\mathrm{H}$ & -1.700897 & 4.903915 & -1.716360 \\
\hline $\mathrm{H}$ & -0.043218 & 4.624912 & -1.176505 \\
\hline $\mathrm{H}$ & 3.210671 & -3.826368 & 3.404876 \\
\hline $\mathrm{H}$ & 2.460180 & -3.491622 & 1.848969 \\
\hline $\mathrm{H}$ & 1.711522 & -4.640124 & 2.962685 \\
\hline $\mathrm{H}$ & -2.932855 & -1.083473 & -0.992414 \\
\hline $\mathrm{H}$ & -4.253941 & -0.672625 & 0.100569 \\
\hline $\mathrm{H}$ & -4.380986 & -2.102192 & -0.923759 \\
\hline $\mathrm{H}$ & 2.039467 & 4.380836 & 1.501877 \\
\hline $\mathrm{H}$ & 0.129070 & -4.844240 & 4.624000 \\
\hline $\mathrm{H}$ & -0.849355 & -3.918300 & 5.767852 \\
\hline $\mathrm{H}$ & 0.864824 & -4.171304 & 6.070994 \\
\hline $\mathrm{H}$ & -1.865336 & 3.491376 & 2.957125 \\
\hline $\mathrm{H}$ & -2.627295 & 1.680443 & -1.212078 \\
\hline $\mathrm{H}$ & -3.026852 & 0.614488 & -2.555886 \\
\hline $\mathrm{H}$ & -3.646186 & 2.265150 & -2.535871 \\
\hline
\end{tabular}




\begin{tabular}{|c|c|c|c|}
\hline & & & \\
\hline & 99 & 79 & \\
\hline & 55 & & \\
\hline & -1.588183 & -1.7 & \\
\hline & -1.2 & & \\
\hline & -0.2 & & \\
\hline & 2.6 & & \\
\hline & -2.21 & & \\
\hline & 3.45 & & \\
\hline & 2.13 & & \\
\hline & -3.7 & & \\
\hline & -4.4 & & \\
\hline & -3.5 & & \\
\hline & 1.51 & -2 & \\
\hline & & & \\
\hline & 3.2 & & \\
\hline & -3.2 & & \\
\hline & -1.8 & & \\
\hline & 3.8 & & \\
\hline & 4.6 & & \\
\hline & 3.8 & & \\
\hline & 1.0 & & \\
\hline & -2.0 & & \\
\hline & -0.9 & & \\
\hline & -1.0 & & \\
\hline & 2.4 & -1. & \\
\hline & 2.1 & & -6 . \\
\hline & 0.8 & -0. & -6 . \\
\hline & -4.0 & & \\
\hline & & & \\
\hline & -2.3 & & \\
\hline & -0.4 & -4 & \\
\hline & -1.1 & -2 . & \\
\hline & -2.0 & & \\
\hline & 5.44 & -0.8 & \\
\hline & $4.3 ?$ & -0.2 & -0.7 \\
\hline & $4.9^{7}$ & 0.8 & -1 . \\
\hline & & & \\
\hline & 3.63 & & -5.4 \\
\hline & & & \\
\hline & -0.981454 & 414 & 40 \\
\hline & & & \\
\hline & & & 4.92 \\
\hline & 0.142568 & 1.412568 & 5.03268 \\
\hline
\end{tabular}




$\begin{array}{cccc}\mathrm{H} & 1.291328 & 1.599116 & 3.711285 \\ \mathrm{H} & 1.865557 & 1.189062 & 5.330809 \\ \mathrm{H} & -0.186182 & -2.132977 & -4.568051 \\ \mathrm{H} & 0.310332 & -3.068655 & -3.155754 \\ \mathrm{H} & 1.102645 & -3.322802 & -4.708721 \\ \mathrm{H} & 2.054370 & 4.357871 & -4.344090 \\ \mathrm{H} & 1.348693 & 5.077080 & -2.897103 \\ \mathrm{H} & 0.658027 & 5.425583 & -4.481314 \\ \mathrm{H} & 0.066129 & 3.469678 & -6.668191 \\ \mathrm{H} & 0.265053 & 1.722294 & -6.745900 \\ \mathrm{H} & 1.579166 & 2.741590 & -6.148889 \\ \mathrm{H} & -2.133230 & -0.254644 & -4.536228 \\ \mathrm{H} & -1.234163 & 0.258435 & -5.965699 \\ \mathrm{H} & -2.772331 & 1.017501 & -5.568576\end{array}$
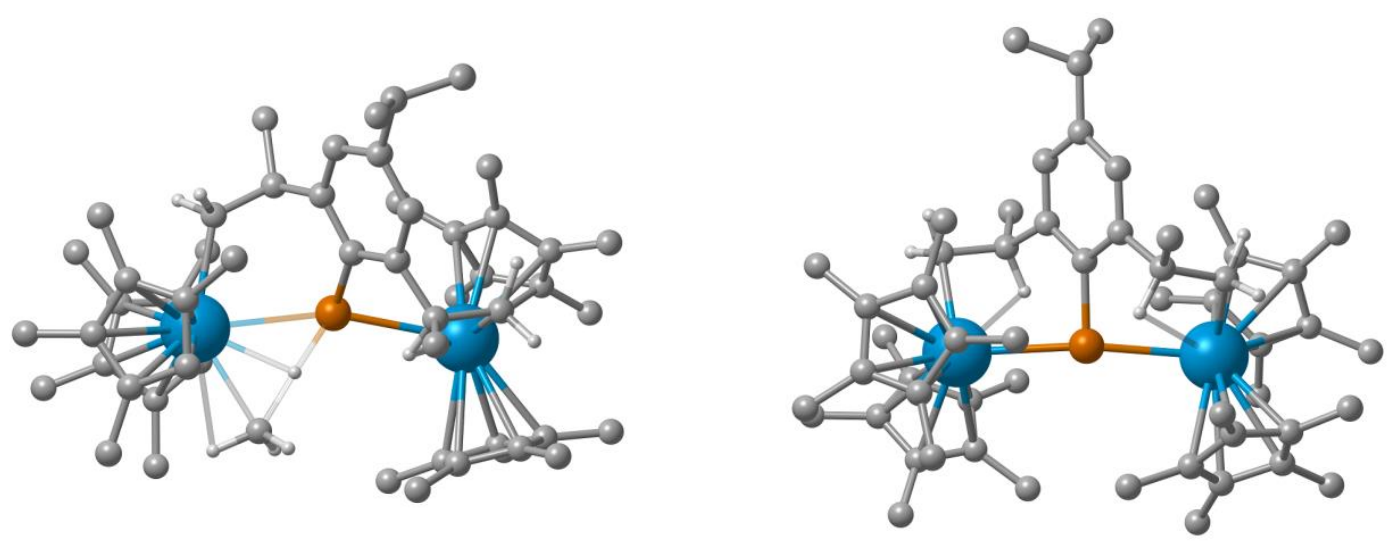

Figure S10. Optimized structures of TS4 (left) and Prod (right). Some methyl carbons and the majority of hydrogen atoms have been omitted for clarity.

\section{$\mathrm{CH}_{4}$}
$\mathrm{E}=-40.508109$
$\mathrm{H}=-40.459215$
$\begin{array}{llll}\text { C } & 0.000000 & 0.000000 & 0.000000\end{array}$
$\begin{array}{llll}\mathrm{H} & 0.000000 & 0.000000 & 1.089000\end{array}$
H $\quad 1.026720 \quad 0.000000 \quad-0.362996$
$\mathrm{H} \quad-0.513360-0.889165 \quad-0.363000$
$\mathrm{H} \quad-0.513360 \quad 0.889165 \quad-0.363000$ 
Table S2. Calculated and experimental geometrical parameters of Prod. Distance and angles are reported in $\AA$ and degrees, respectively.

\begin{tabular}{|l|c|c|}
\hline & Calculated structure & Experimental structure \\
\hline Th-P distance & 2.89 and 2.86 & $2.82(1)$ and 2.81(1) \\
\hline P-C distance & 1.91 & $1.87(1)$ \\
\hline Th-CH $\mathrm{CH}_{2}$ distance & 2.46 and 2.49 & $2.47(1)$ and $2.48(1)$ \\
\hline Th-P-Th angle & 170.2 & $174.0(1)$ \\
\hline $\mathrm{CH}_{2}-\mathrm{Th}-\mathrm{P}$ angle & 97.2 and 88.5 & $95.2(1)$ and $93.5(1)$ \\
\hline
\end{tabular}

\section{References}

(1) Cantat, T.; Scott, B. L.; Kiplinger, J. L. Chem. Commun. 2010, 46, 919.

(2) Fagan, P. J.; Manriquez, J. M.; Maatta, E. A.; Seyam, A. M.; Marks, T. J. J. Am. Chem. Soc. 1981, 103, 6650.

(3) Chandrasekhar, V.; Sasikumar, P.; Boomishankar, R.; Anantharaman, G. Inorg. Chem. 2006, 45, 3344.

(4) van den Winkel, Y.; Bastiaans, H. M. M.; Bickelhaupt, F. J. Organomet. Chem. 1991, 405, 183.

(5) Gaussian 09, Revision D.01, Frisch, M. J.; Trucks, G. W.; Schlegel, H. B.; Scuseria, G. E.; Robb, M. A.; Cheesman, J. R.; Scalmani, G.; Barone, V.; Mennucci, B.; Petersson, G. A.; Nakatsuji, H.; Caricato, M.; Li, X.; Hratchian, H. P.; Izmaylov, A. F.; Bloino, J.; Zheng, G.; Sonnenberg, J. L.; Hada, M.; Ehara, M.; Toyota, K.; Fukuda, R.; Hasegawa, J.; Ishida, M.; Nakajima, T.; Honda, Y.; Kitao, O.; Nakai, H.; Vreven, T.; Montgomery, J. A., Jr.; Peralta, J. E.; Ogliaro, F.; Bearpark, M.; Heyd, J. J.; Brothers, E.; Kudin, K. N.; Staroverov, V. N.; Kobayashi, R.; Normand, J.; Raghavachari, K.; A., R.; Burant, J. C.; Iyengar, S. S.; Tomasi, J.; Cossi, M.; Rega, N.; Millam, M. J.; Klene, M.; Knox, J. E.; Cross, J. B.; Bakken, V.; Adamo, C.; Jaramillo, J.; Gomperts, R.; Stratmann, R. E.; Yazyev, O.; Austin, A. J.; Cammi, R.; Pomelli, C.; Ochterski, J. W.; Martin, R. L.; Morokuma, K.; Zakrzewski, V. G.; Voth, G. A.; Salvador, P.; Dannenberg, J. J.; Dapprich, S.; Daniels, A. D.; Farkas, O.; Foresman, J. B.; Ortiz, J. V.; Cioslowski, J.; Fox, D. J., Gaussian Inc., 2009, Wallingford CT

(6) Moritz, A.; Cao, X.; Dolg, M. Theor. Chem. Acc. 2007, 118, 845.

(7) Hehre, W. J.; Ditchfield, R.; Pople, J. A. J. Chem. Phys. 1972, 56, 2257.

(8) Bergner, A.; Dolg, M.; Küchle, W.; Stoll, H.; Preuß, H. Mol. Phys. 1993, 80, 1431.

(9) Becke, A. D. J. Chem. Phys. 1993, 98, 5648.

(10) Burke, K.; Perdew, J. P.; Yang, W. Electronic Density Functional Theory: Recent Progress and New Directions; Plenum: New York, 1998.

(11) Reed, A. E.; Curtiss, L. A.; Weinhold, F. Chem. Rev. 1988, 88, 899.

(12) APEX2 Suite, Bruker AXS Inc, 2006, Madison, WI

(13) Sheldrick, G. Acta Cryst. 2015, 71, 3.

(14) Barbour, L. J. Supramol. Chem. 2001, 1, 189. 